\title{
Effectiveness and safety of dipeptidyl peptidase 4 inhibitors in the management of type 2 diabetes in older adults: a systematic review and development of recommendations to reduce inappropriate prescribing
}

Gisela Schott $^{1 *+}$, Yolanda V Martinez ${ }^{2 \dagger}$, R. Erandie Ediriweera de Silva ${ }^{2,3}$, Anna Renom-Guiteras ${ }^{4,5}$, Anna Vögele ${ }^{6}$, David Reeves ${ }^{2}$, Ilkka Kunnamo ${ }^{7}$, Minna Marttila-Vaara ${ }^{7}$ and Andreas Sönnichsen ${ }^{4}$

\begin{abstract}
Background: Preventable drug-related hospital admissions can be associated with drugs used in diabetes and the benefits of strict diabetes control may not outweigh the risks, especially in older populations. The aim of this study was to look for evidence on risks and benefits of DPP-4 inhibitors in older adults and to use this evidence to develop recommendations for the electronic decision support tool of the PRIMA-eDS project.
\end{abstract}

Methods: Systematic review using a staged approach which searches for systematic reviews and meta-analyses first, then individual studies only if prior searches were inconclusive. The target population were older people ( $\geq 65$ years old) with type 2 diabetes. We included studies reporting on the efficacy and/or safety of DPP-4 inhibitors for the management of type 2 diabetes. Studies were included irrespective of DPP-4 inhibitors prescribed as monotherapy or in combination with any other drug for the treatment of type 2 diabetes. The target intervention was DPP-4 inhibitors compared to placebo, no treatment, other drugs to treat type 2 diabetes or a non-pharmacological intervention.

Results: Thirty studies (reported in 33 publications) were included: 1 meta-analysis, 17 intervention studies and 12 observational studies. Sixteen studies were focused on older adults and 14 studies reported subgroup analyses in participants $\geq 65, \geq 70$, or $\geq 75$ years. Comorbidities were reported by 26 studies and frailty or functional status by one study. There were conflicting findings regarding the effectiveness of DPP-4 inhibitors in older adults. In general, DPP-4 inhibitors showed similar or better safety than placebo and other antidiabetic drugs. However, these safety data are mainly based on short-term outcomes like hypoglycaemia in studies with HbA1c control levels recommended for younger people. One recommendation was developed advising clinicians to reconsider the use of DPP-4 inhibitors for the management of type 2 diabetes in older adults with $\mathrm{HbA} 1 \mathrm{c}<8.5 \%$ because of scarce data on clinically relevant benefits of their use. Twenty-two of the included studies were funded by pharmaceutical companies and authored or co-authored by employees of the sponsor.

\footnotetext{
* Correspondence: gisela.schott@akdae.de

${ }^{\dagger}$ Equal contributors

'Drug Commission of the German Medical Association, Berlin, Germany

Full list of author information is available at the end of the article
} 
(Continued from previous page)

Conclusions: Other than the surrogate endpoint of improved glycaemic control, data on clinically relevant benefits of DPP-4 inhibitors in the treatment of type 2 diabetes mellitus in older adults is scarce. DPP-4 inhibitors might have a lower risk of hypoglycaemia compared to other antidiabetic drugs but data show conflicting findings for long-term benefits. Further studies are needed that evaluate the risks and benefits of DPP-4 inhibitors for the management of type 2 diabetes mellitus in older adults, using clinically relevant outcomes and including representative samples of older adults with information on their frailty status and comorbidities. Studies are also needed that are independent of pharmaceutical company involvement.

Keywords: Systematic review, Dipeptidyl-peptidase IV inhibitors, Type 2 diabetes mellitus, Inappropriate prescribing

\section{Background}

Diabetes is a prevalent chronic disease worldwide. The International Diabetes Federation estimated the prevalence of diabetes to be $8.8 \%$ in adults 20 to 79 years old and close to $20 \%$ in people aged over 65 years [1]. Diabetes and its complications are an important cause of morbidity and mortality, and people with diabetes have substantially reduced life expectancy [2]. Duration of diabetes and the degree of metabolic control are important factors determining the prognosis for people with diabetes [3]. However, drugs used in diabetes are one of the most commonly used drug groups associated with preventable hospital admissions related to adverse drug events and overtreatment, especially in older populations [4]. Furthermore, some studies suggest that strict metabolic control may not be advisable for older and frail people, because the benefits may not outweigh the risks of the treatment [5].

Dipeptidyl peptidase-4 (DPP-4) inhibitors are oral agents used for the pharmacological treatment of adults with type 2 diabetes mellitus. The main representatives of this class are sitagliptin, saxagliptin, vildagliptin, linagliptin, teneligliptin and alogliptin.

DPP-4 is a protease involved in glucagon-like peptide-1 (GLP-1) inactivation. By inhibiting the enzyme, DPP-4 inhibitors prolong and enhance the activity of GLP-1 [6]. GLP-1 exerts its main effects by stimulating glucosedependent insulin release, slowing gastric emptying, reducing food intake, and decreasing postprandial glucagon excretion.

The approved indications for DPP-4 inhibitors are limited to patients for whom diet and exercise do not provide adequate glycaemic control. In addition, first line use of metformin is recommended unless metformin is not tolerated or contraindicated [7], and this is also the case for older populations [8]. In clinical guidelines, DPP-4 inhibitors are recommended only as a second or third line treatment $[7,9,10]$.

A systematic review has shown that in patients with type 2 diabetes, who do not achieve the glycaemic targets with metformin alone, DPP-4 inhibitors can lower $\mathrm{HbA1c}$ to the same extent as sulfonylureas or pioglitazone, with neutral effect on body weight [11]. However, this systematic review did not report the age ranges of the participants in the included studies. Furthermore, HbA1c and body weight are arguably only surrogate outcomes for more clinically relevant endpoints such as physical and mental status, quality of life, and life expectancy.

Data on long-term risks and benefits of DPP4inhibitors are scarce. Only three randomised controlled trials of DPP4-inhibitors have looked at clinically relevant endpoints for an observation period of at least 18 months [12-14]. These trials respectively compared saxagliptin, sitagliptin and alogliptin to placebo (alongside existing therapy) [12-14]. However, all these trials report only minimal, or no, results specific to older participants (65 years or more) [12-14]. This reflects the common problem that older people, despite being major users and potentially having a different response to pharmaceutical interventions, are under-represented in most drug trials $[15,16]$, and that clinical guidelines often base their recommendations on evidence mostly from younger populations [17]. To the best of our knowledge, no systematic review has evaluated the specific evidence on the use of DPP-4 inhibitors in older populations.

The objectives of this systematic review (SR) are therefore:

- to systematically review the literature on the risks and benefits of the use of DPP-4 inhibitors in the treatment of type 2 diabetes in older adults,

- to critically assess the quality of the evidence identified, and

- to develop recommendations in relation to discontinuation or dose-adjustment of DPP-4 inhibitors in the treatment of type 2 diabetes in older adults.

The recommendations developed will be used in an electronic decision support tool in the PRIMA-eDS project [18]. 


\section{Methods}

This SR was conducted following an adaption of the methods recommended by both the Cochrane Handbook for Systematic Reviews of Interventions [19] and the Preferred Reporting Items for Systematic Reviews and Meta-Analyses (PRISMA) [20].

For undertaking this SR, as one of a planned longterm series of SRs on the efficacy and safety of commonly prescribed drugs in older people, we purposely developed an efficient methodology that does not compromise quality. A full description of our methods has been published [21], but in brief we developed a fourstage approach by which we initially search for systematic reviews and meta-analyses (search 1 and 2) and only if necessary move on to searching for individual studies (search 3A and 3B; see Search method below). Each subsequent stage is only undertaken if the accumulated evidence from the previous stages is deemed not sufficient, or of sufficient quality, to enable evidence based recommendations to be made. A specific protocol for the present SR was prepared and is available from the authors upon request.

\section{Study inclusion criteria Types of studies}

In line with our methodology, in a staged fashion we included systematic reviews, meta-analyses, controlled interventional studies and observational studies reporting on risks and benefits of the use of DPP-4 inhibitors in the treatment of type 2 diabetes in older adults. We excluded conference abstracts, pooled analyses, editorials, opinion papers, case reports, case series, narrative reviews, letters, and qualitative studies.

\section{Type of participants}

We explicitly searched for studies on older people ( $\geq 65$ years old) with type 2 diabetes. Our specific age criteria for inclusion varied according to study design:

For systematic reviews and meta-analyses (any of the following criteria):

- Overall mean or median age $\geq 65$ years;

- Overall mean or median age < 65 but subgroup analysis reporting on participants $\geq 65$ years;

- Overall mean or median age not reported but $80 \%$ or more of the included studies reported a mean or median age $\geq 65$ years.

For controlled interventional studies and observational studies (any of the following criteria):

- $\geq 80 \%$ of participants $\geq 65$ years;

- $<80 \%$ of participants $\geq 65$ years but subgroup analysis reporting on participants $\geq 65$ years.

\section{Types of interventions}

We included studies reporting on the efficacy and/or safety of any DPP-4 inhibitor for the management of type 2 diabetes. Studies were included irrespective of DPP-4 inhibitors prescribed as monotherapy or in combination with any other drug for the treatment of type 2 diabetes. We included studies comparing DPP-4 inhibitors versus placebo, no treatment, other drugs to treat type 2 diabetes or a non-pharmacological intervention.

\section{Types of outcomes}

We included studies that used any of the following clinically relevant endpoints as primary or secondary outcomes: hypoglycaemia, adverse events, quality of life, mortality, life expectancy, a related hospitalisation, cognitive impairment or cognitive status, functional impairment or functional status, cardiovascular events including stroke, renal failure, composite end points including any of the above, any of the above evaluated as safety endpoints. Studies reporting other outcomes considered as clinically relevant were also considered for inclusion. We excluded studies evaluating only glycaemic control, changes in HbA1c levels or other endpoints considered to be not clinically relevant. To aid interpretation of findings we have classified outcomes into two tiers according to their anticipated impact on longerterm health and quality of life: Tier 1 outcomes generally have shorter-term impact and include hypoglycaemia and adverse events (including serious adverse events); Tier 2 outcomes have longer-term impact and include, but aren't limited to, cardiovascular and cerebrovascular events, related hospitalisations, and death.

\section{Setting}

We included any setting reporting on the management of type 2 diabetes using DPP-4 inhibitors.

\section{Language}

We did not apply any language restriction to the search but we only included studies that could be read by the research team (languages: English, German, Finish, Italian, and Spanish).

\section{Search method}

Database searches were conducted by YVM. We started searching for systematic reviews and meta-analyses (search 1 and 2). During study selection under search 1 and 2, we identified eligible individual studies from excluded systematic reviews and meta-analyses and transferred these to the Search 3A list for potential inclusion. The list of studies in Search 3A was checked for inclusion following the procedures described below under "Selection of studies". Only one relevant meta-analysis was found from Searches 1 and 2. However, this meta- 
analysis covered just one type of DPP-4 inhibitor (linagliptin). Therefore, we conducted Search 3B for individual studies published in the last 10 years (2005-2015) [21]. Detailed information about databases and search dates is summarised below:

- Search 1 was conducted on 03 December 2015 in the Cochrane Database of Systematic Reviews (OVID interface, 2005 to November 2015) and the Database of Abstracts or Reviews of Effects (DARE, OVID interface, 1991 to 2nd Quarter 2015).

- Search 2 was conducted on 03 December 2015 in MEDLINE (OVID interface, 1946 to November Week 3 2015), EMBASE (OVID interface, 1974 to 2015 December 02), Health Technology Assessment (HTA, OVID interface 2001 to 4th Quarter 2015) and International Pharmaceutical Abstracts (IPA, OVID interface 1970 to November 2015).

- Search 3A consisted of controlled intervention and observational studies from systematic reviews and meta-analysis not included in searches 1 and 2 but containing eligible studies.

- Search 3B was conducted on 7 December 2015 in MEDLINE (OVID interface, 2005 to November Week 3 2015), EMBASE (OVID interface, 2005 to 2015 December 04), HTA (OVID interface 2005 to 4th Quarter 2015) and IPA (OVID interface 2005 to November 2015).

In addition to database searches, we checked the references of included reviews and studies following the procedures described later under "Selection of studies". A list of excluded studies after full-text check with reasons for exclusion is provided in Additional file 1.

The PICOS-framework was used to develop the search terms (population: older people with type 2 diabetes, intervention: DPP-4 inhibitors, comparison: any, outcomes: see list above "Types of outcomes" and study design: systematic reviews, meta-analyses, controlled interventional studies and observational studies). We also created search filters specific to different study designs and each filter is described in detail in the protocol [21]. Additional file 2 shows the full search terms for each search (i.e. Searches 1, 2 and 3B).

\section{Data management}

Literature search results were uploaded to the Endnote X7 reference management software. Endnote was used to import search results and to de-duplicate references.

\section{Selection of studies}

First, two independent reviewers assessed titles and abstracts from each search and identified studies to include. Second, full manuscripts were obtained for all titles and abstracts that met the inclusion criteria or where there was any uncertainty for inclusion. GS, AV, YVM and REED were involved in this task. Reviewers agreed on which articles should be included and ARG acted as arbitrator when GS, AV, YVM and REED could not reach a full consensus.

\section{Data extraction}

GS, YVM and REED independently conducted data extraction from each study using a standardised and piloted data collection form which has been published alongside the protocol [21]. GS, YVM and REED checked each other's data extraction to look for completeness and accuracy. The data extraction form collected information related to the study design and aim, characteristics of participants (age, sex, setting, comorbidities, use of concomitant medications, functional status, frailty, and cognitive status), the intervention (i.e. DPP-4 inhibitors) and comparison, time to followup, and reported outcomes. We also collected information on the involvement of pharmaceutical companies in the included studies.

\section{Quality appraisal}

We used three validated assessment tools to assess the quality of the evidence from each included study: for systematic reviews/meta-analyses the Assessment of Multiple Systematic Reviews tool (AMSTAR) [22, 23], for intervention studies the Cochrane Collaboration's tool for assessing risk of bias [19], and for observational studies the Critical Appraisal Skills Programme (CASP) [24, 25].

An overall rating for each study was made based on study limitations as suggested by Guyatt et al. (2008) [26], starting with high quality for randomised trials without important limitations (such as lack of allocation concealment; lack of blinding, large loss at follow-up, unmet intention to treat analysis, stopping early for benefit; and failure to report outcomes) and low quality for observational studies without important limitations.

\section{Dealing with duplicate and companion publications}

We included all relevant data from publications relating to a single primary study. Due to our staged approach, it was possible that a publication that was part of an included systematic review or meta-analysis, would also be included as a separate individual study, resulting in a risk of "double-counting". Any such instances have been identified and reported and taken account of in our synthesis of results.

\section{Data synthesis}

A narrative synthesis describing all included systematic reviews, meta-analyses, intervention and observational studies, participants and findings was carried out. The 
included studies were highly heterogeneous regarding type of DPP-4 inhibitors, comparison (form of control treatment or placebo), length of follow-up and outcome definition (e.g. types of adverse events included); therefore no additional meta-analyses were performed. The quality of the included studies is also reported.

\section{Identification of "references of interest" for the development of recommendations}

During the search process, GS, and YVM identified and collected additional material relevant to the development of recommendations according to the methodology described by Martinez/Renom - Guiteras et al. (2017) [21].

\section{Development of recommendations}

Included studies and additional references were summarised in a document that was used in team meetings to develop recommendations on when the use of DPP-4 inhibitors could be safely discontinued or the dosage reduced in the management of type 2 diabetes in older people [21]. Each recommendation was given a rating for strength (weak or strong) and quality (low, moderate or high) of evidence following the GRADE methodology [26-28].

\section{Results}

\section{Results of the search}

We identified 1460 records through initial database searching (21 from search 1, 82 from search 2, 9 from search 3A and 1357 from search 3B). Additionally, we identified 988 records from reference lists of included studies, and one further study by snowballing. After removing duplicates, we screened 2009 records and excluded 1634 records after checking titles and abstracts. We assessed 375 full-texts for eligibility and excluded 341 records. We included 30 studies reported in 33 publications. The PRISMA flow diagram is presented in Fig. 1.

\section{Included studies}

Table 1 shows details of included studies. Thirty studies met our inclusion criteria. These studies included more than 273,358 participants $\geq 65$ years. The largest had 141,322 participants and the smallest 60 participants.

\section{Study designs}

Seventeen of the included studies were interventional designs, one was an MA and 12 were observational in nature. None of the individual studies were also part of the MA. Length of follow-up varied from 12 weeks to 5 years. Data on outcomes was extracted at the end of follow-up for each included study. In 16 out of 30 studies information was given about the countries where studies had been conducted: the USA [29, 30], Australia, Canada, Denmark, the Netherlands and Sweden [31], Taiwan [32-36], 38 countries [13], 13 countries [37], Japan [38], 12 European countries and Mexico [39], 14 European countries [40], 26 countries [12, 41-43], 49 countries [44], UK [45-47], Italy [48], France [49], Spain [50], and Greece [51].

\section{Participants}

Table 1 shows included studies involving older adults (at least $80 \%$ people $\geq 65$ years: 16 studies) or presenting subgroup analyses in participants $\geq 65$ years (11 studies including the meta-analysis), $\geq 75$ years ( 2 studies), and $\geq 70$ years ( 1 study). Additional file 3: Table S1 shows the characteristics of the participants in the included studies. Age is reported as mean or median years; for the whole sample where available, else for the different treatment groups. Mean age was reported in 27 studies and ranged from 53.1 to 80.2 years. Median age was reported in 3 studies and ranged from 58 to 77 years.

All included studies reported on participant sex (30 studies), though in some cases by treatment group only. The percentage of male participants ranged from $36.7 \%$ to $71.6 \%$.

Fourteen studies reported ethnicity with the most common classification being white (range: 53.9 to 98.6\%). Information about the care setting was reported by five studies: primary care in the USA [29], primary care in the UK [45], primary care in France [49], hospital department of internal medicine in Greece [51], and primary and hospital care in the UK [47]. Information about comorbidities was provided by 26 out of 30 studies. Concomitant diseases were frequent and hypertension and dyslipidaemia most commonly reported. Eighteen studies reported on concomitant medications with a majority of patients taking antihypertensive and lipid-lowering medications. Frailty status was reported by one study [52], with about $10 \%$ of patients assessed as frail. One study reported on disability after stroke as the main outcome, but no baseline data on disability were provided [51]. Cognitive status was not reported by any of the studies.

\section{Interventions and outcomes}

Most of the included studies addressed only our lower tier endpoints: adverse events and hypoglycaemia. A minority of studies investigated Tier 2 outcomes such as death, hospitalisation, cardiovascular events and, in one case, functional status. We found no studies in older people reporting on the clinically relevant endpoints of: quality of life, life expectancy, cognitive impairment or cognitive status.

\section{Vildagliptin}

Vildagliptin (50 or $100 \mathrm{mg} /$ daily) was examined in 9 out of 30 studies. Vildagliptin was compared with placebo in two trials $[52,53]$, with glimepiride in two trials $[54,55]$, with metformin in one trial [40] and with 


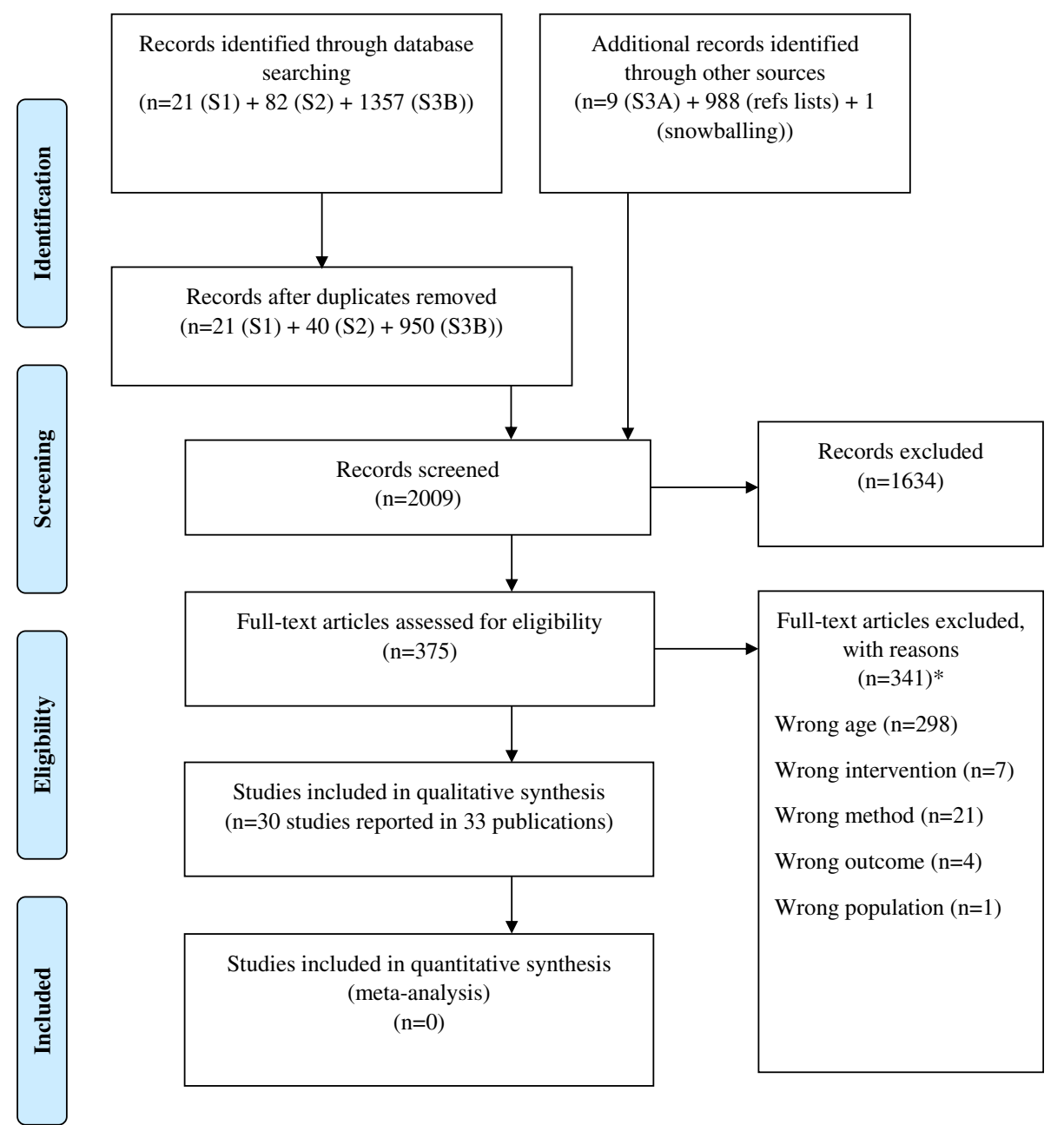

Fig. 1 Preferred Reporting Items for Systematic Reviews and Meta-Analyses (PRISMA) flow diagram. *Additional file 1 includes the list of excluded studies with reasons

thiazolidinediones in one trial [29]. Also included were one uncontrolled trial on vildagliptin [56] and two observational studies [50,57]. The outcomes for these studies were adverse events $[29,40,52-56]$, serious adverse events [52, 54-56], hypoglycaemia [40, 50, 52-57] and a list of other outcomes by Sicras-Mainar and Navarro-Artieda (2014) (macrovascular complications and cardiovascular events, cerebrovascular disease, all types of peripheral arterial disease and renal disease) [50].

\section{Sitagliptin}

Sitagliptin (25 to $100 \mathrm{mg} /$ daily) was examined in five studies: two placebo-controlled [13, 30], one uncontrolled [32], one active controlled [37], and one cohort study [34]. Three of these studies included adverse events as one of their outcomes and their primary endpoint was change in HbA1c [30, 34, 37]. The other two studies reported a composite of cardiovascular events as their primary endpoints $[13,34]$.

\section{Linagliptin}

Linagliptin (5 $\mathrm{mg} /$ daily) was compared with placebo in one trial [31]. The outcomes were adverse events, hypoglycaemia, and cardiovascular events [31]. Also, one meta-analysis investigated the cardiovascular safety of linagliptin [58].

\section{Teneligliptin}

Teneligliptin (20 mg/daily) was compared to placebo in one study [38], with adverse events and hypoglycaemia as outcomes.

\section{Alogliptin}

Alogliptin (25 mg/daily) was compared to glipizide in one study [59] and to placebo in another study [44]. Adverse events and hypoglycaemia were the outcomes in 


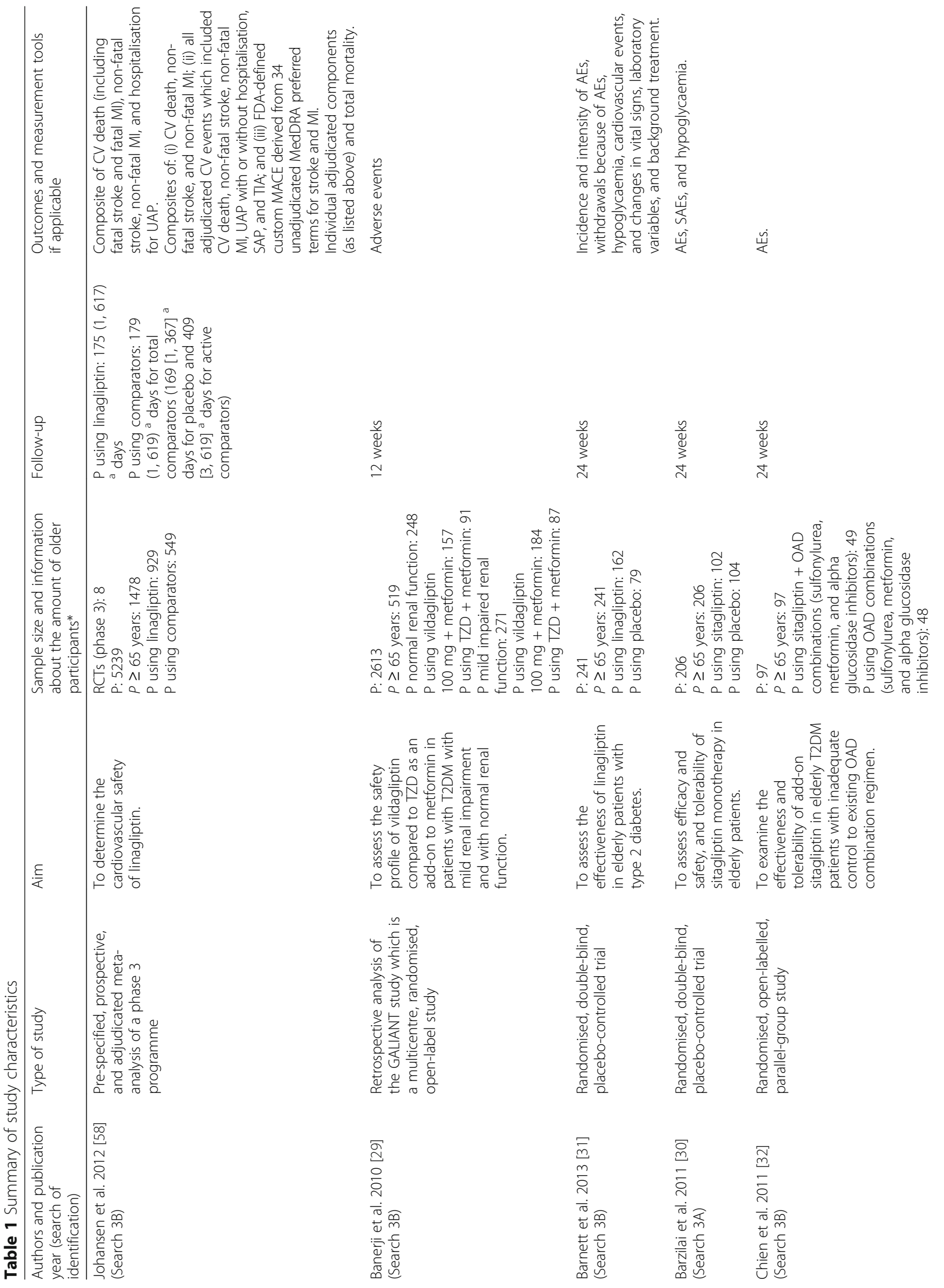



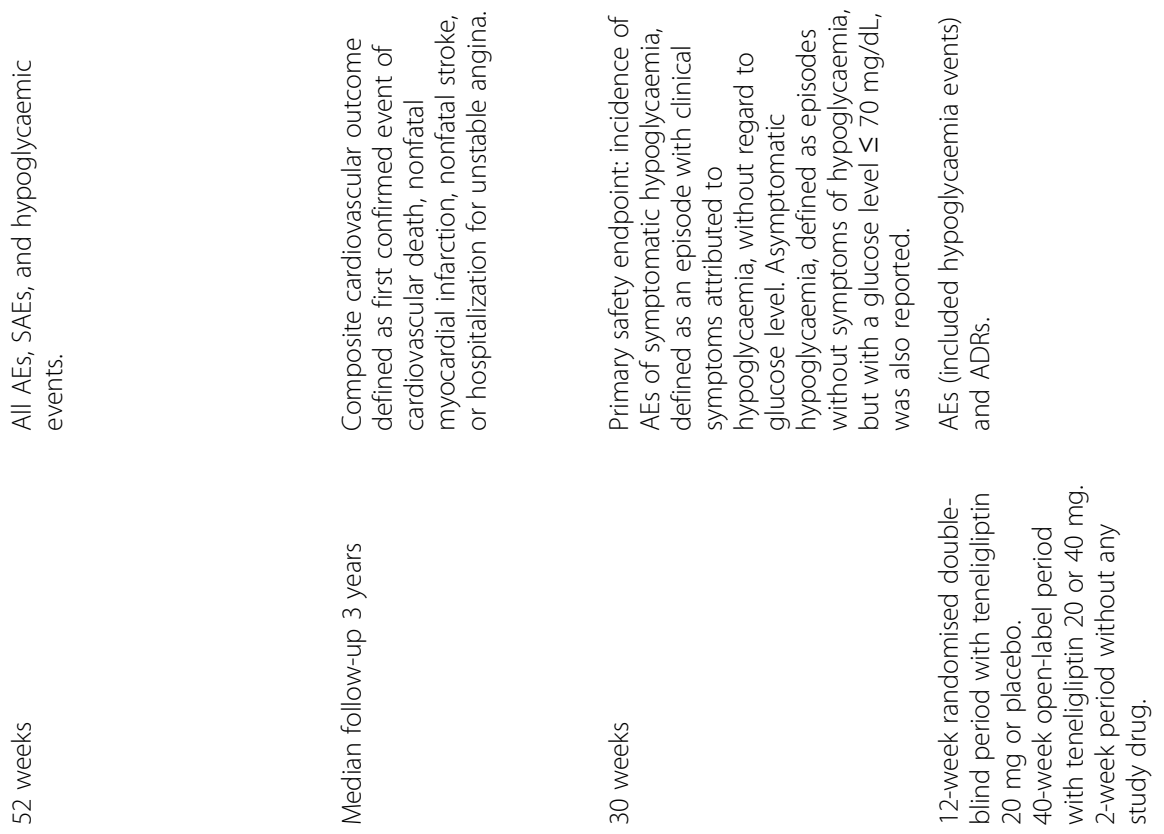

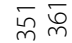
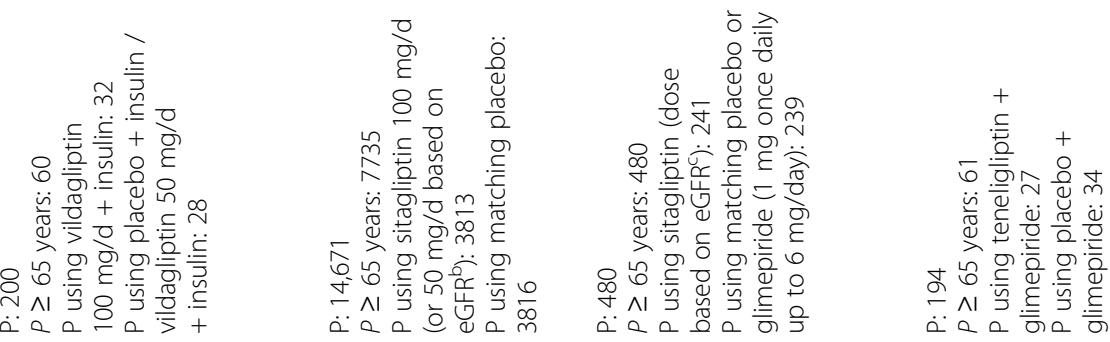

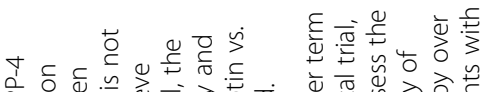

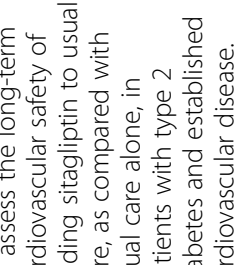

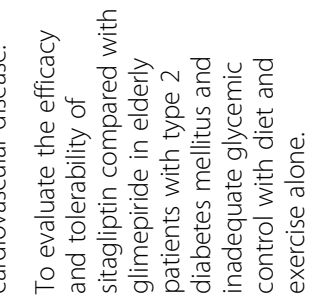

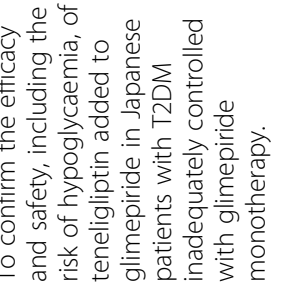

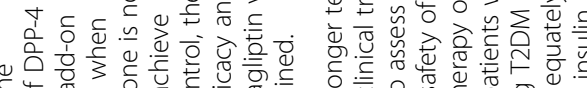

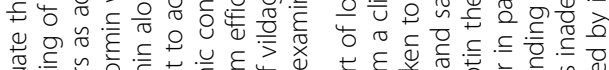

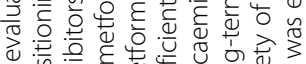

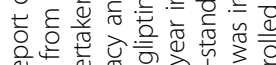

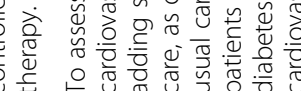
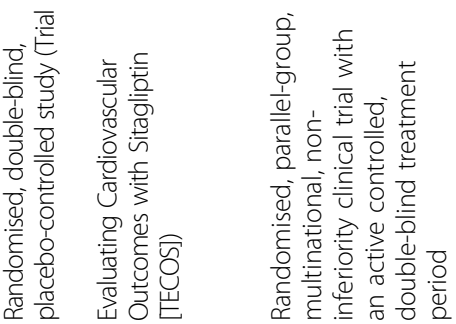

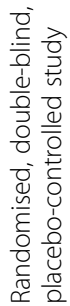

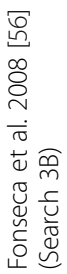

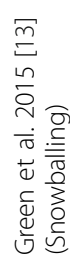

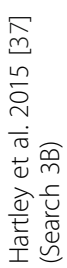

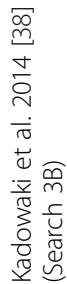




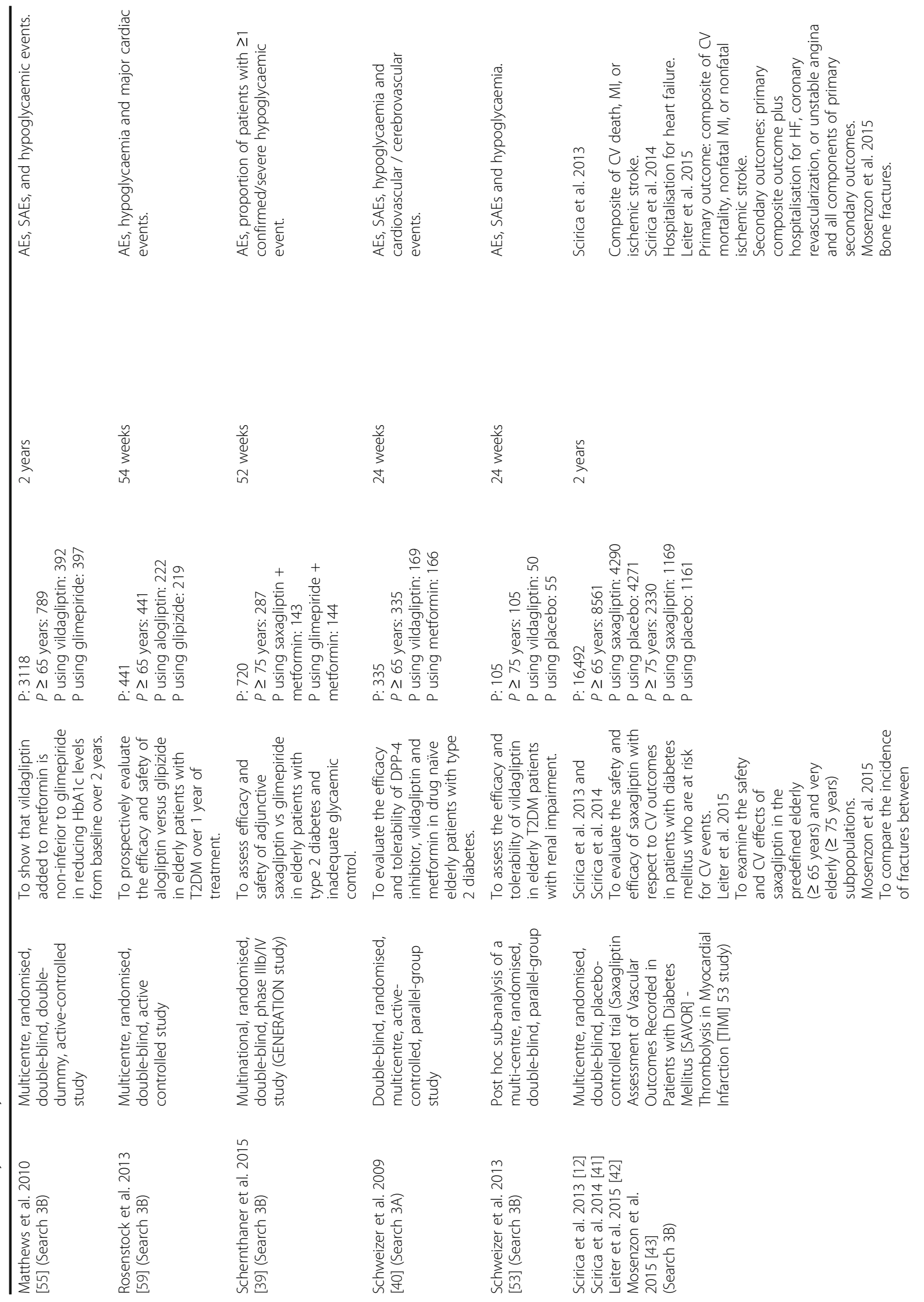




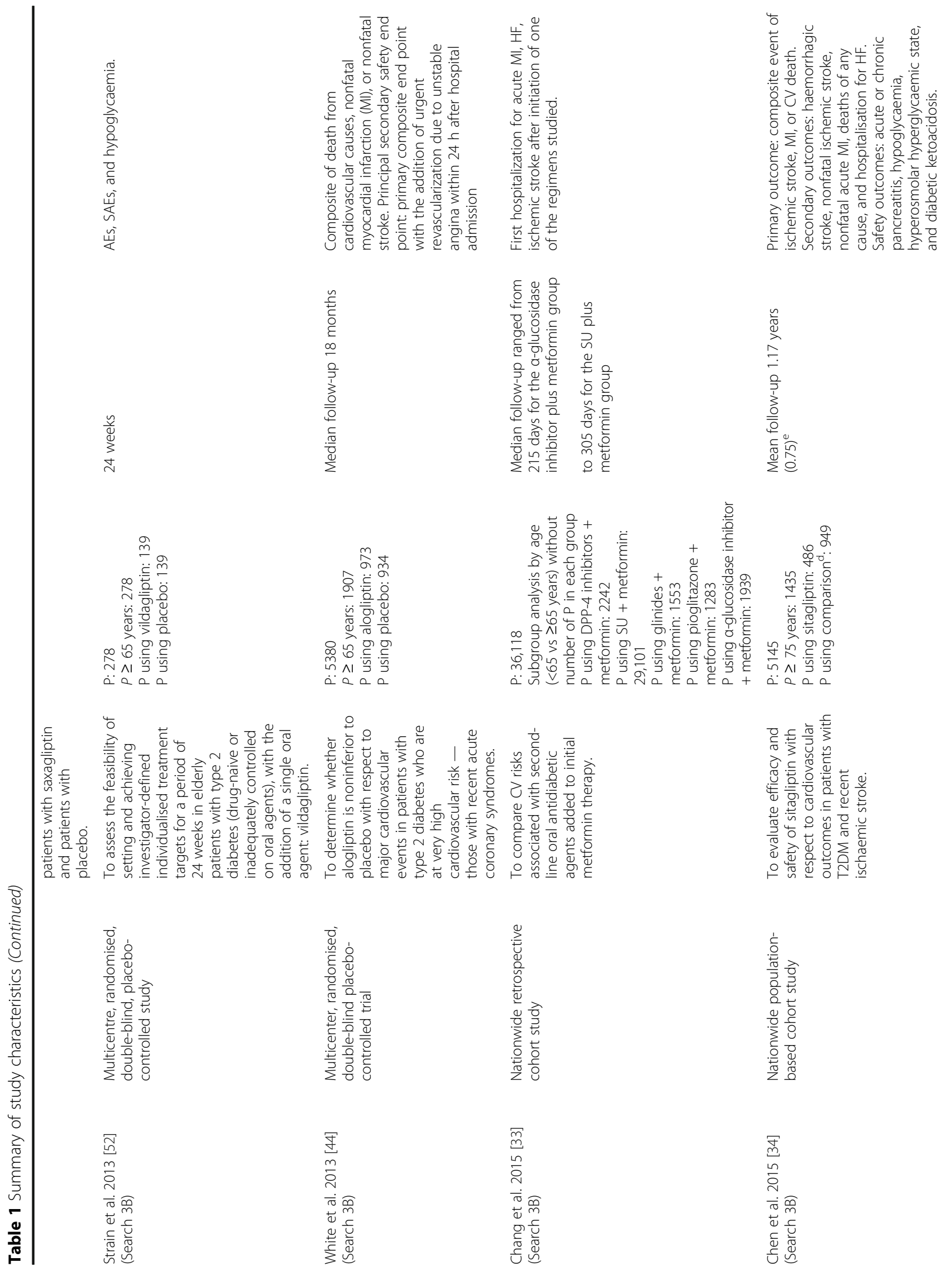



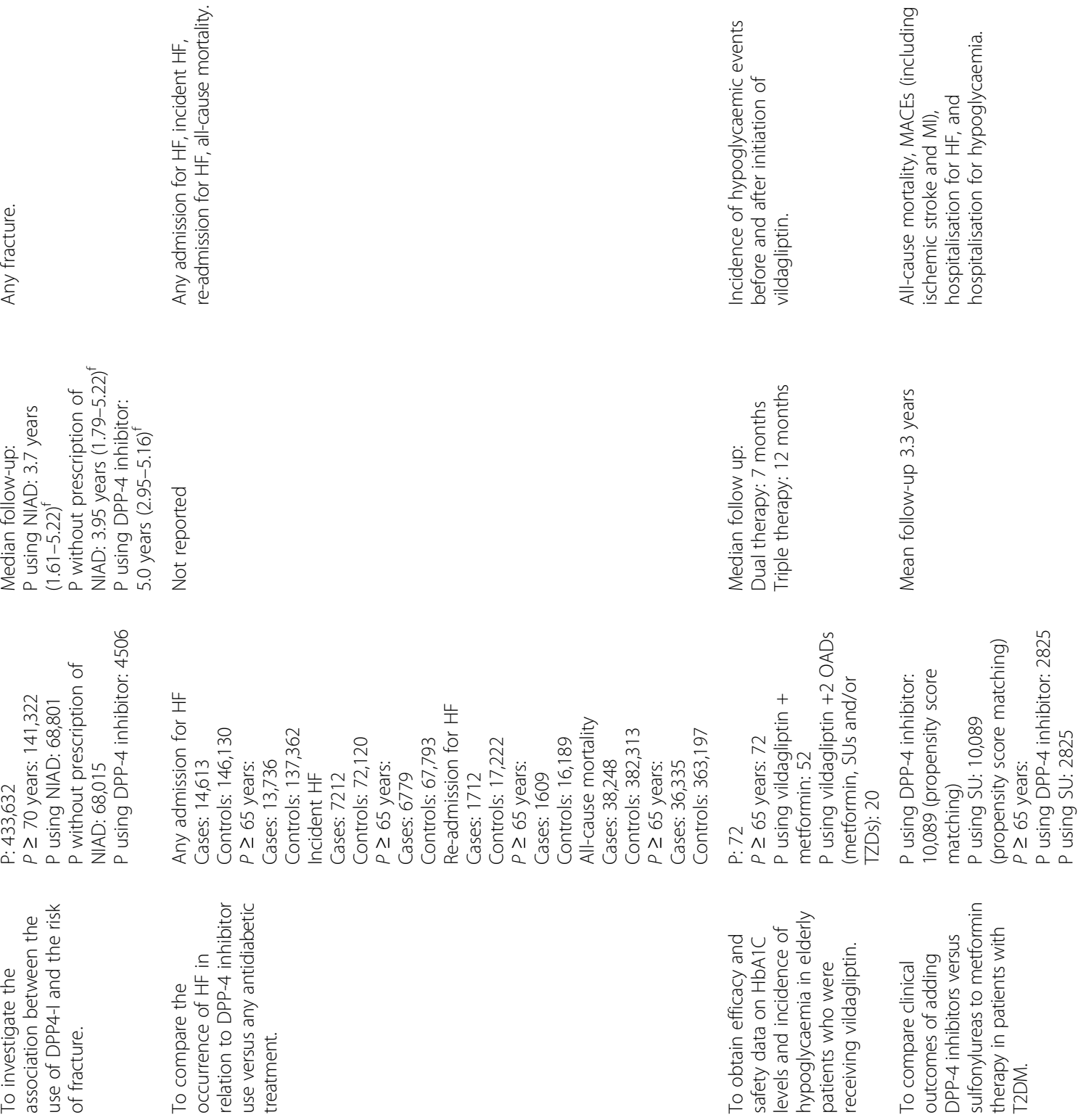

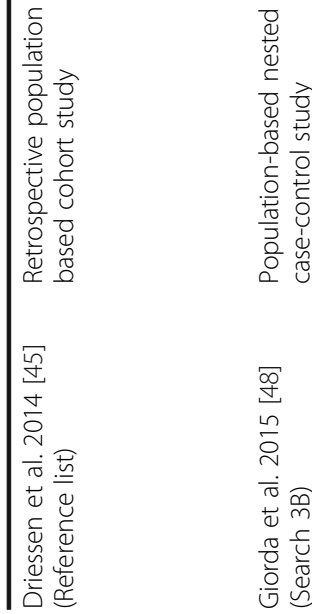

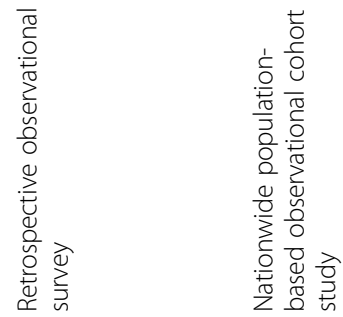

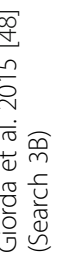

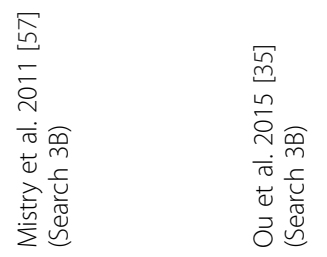




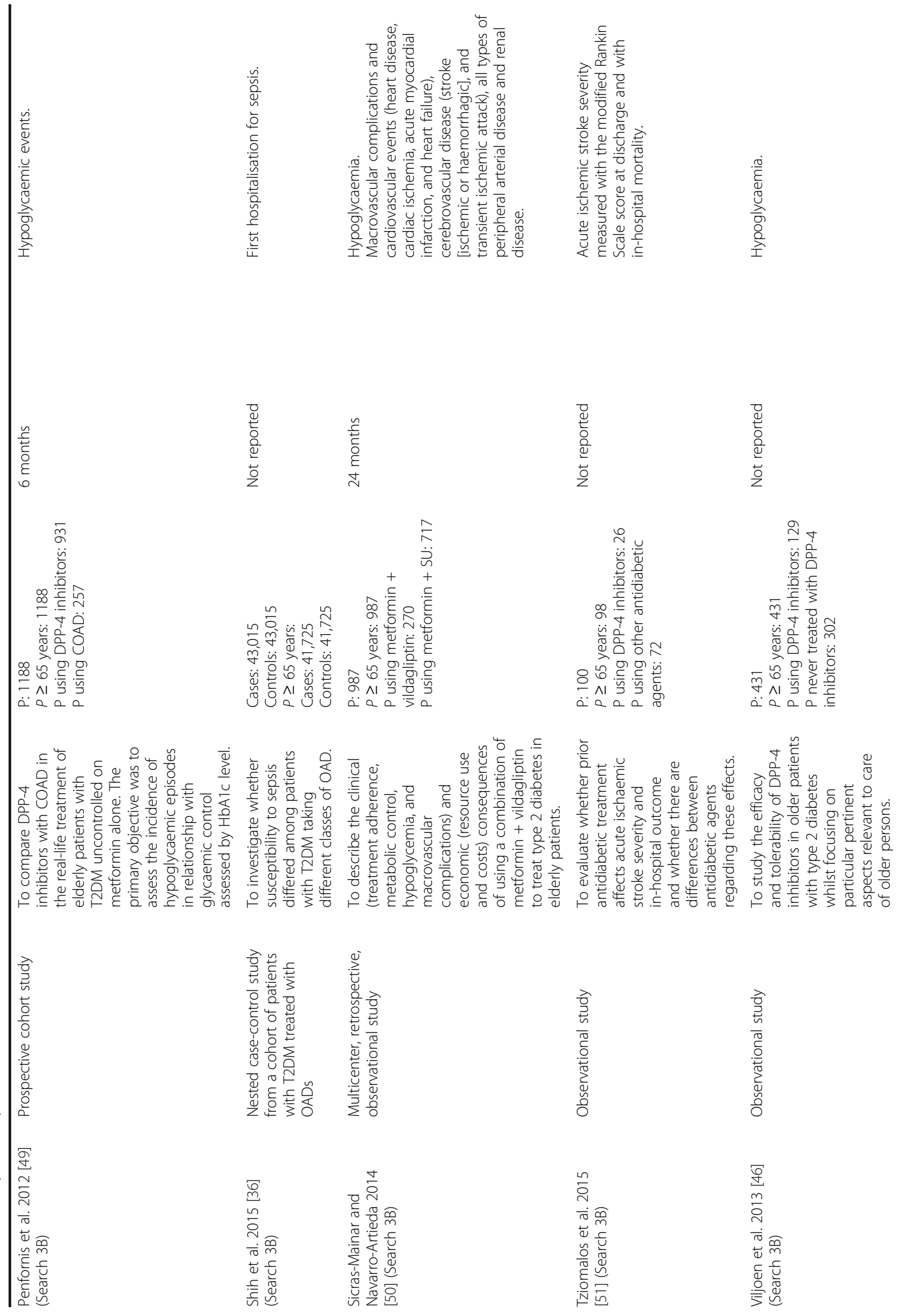




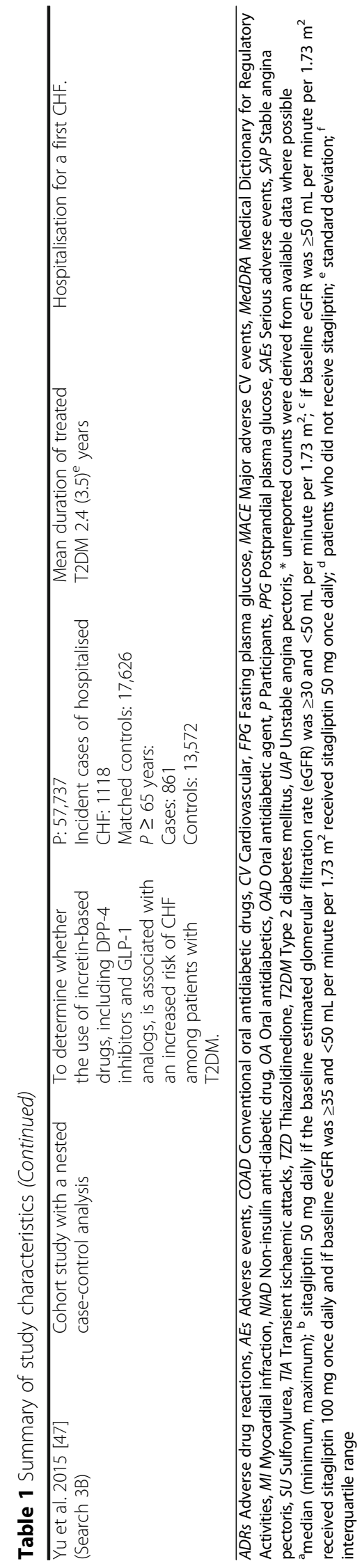


one study [59]. The other study used a composite outcome of death from cardiovascular causes, nonfatal myocardial infarction, or nonfatal stroke [44].

\section{Saxagliptin}

Saxagliptin (5 mg/daily) was compared to placebo in one study reported by four publications with the following outcomes: a composite outcome of cardiovascular death, myocardial infarction, or ischemic stroke [41], hospitalisation for heart failure [12], a composite outcome of cardiovascular mortality, nonfatal myocardial infarction, or nonfatal ischemic stroke with and without hospitalisation for heart failure, coronary revascularization, or unstable angina as well as the individual components [42], and bone fractures [43]. One randomised trial compared saxagliptin ( $5 \mathrm{mg} /$ daily) against glimepiride $(1 \mathrm{mg} /$ daily) with hypoglycaemia and adverse events as safety outcomes and glycaemic control as the primary outcome.

\section{Any DPP-4 inhibitor}

Nine observational studies compared patients treated with DPP-4 inhibitors with patients not receiving DPP-4 inhibitors [46]; other antidiabetic drugs [33, 35, 45, 49, 51]; or between cases and controls [36, 47, 48]. These studies reported on the following outcomes: hypoglycaemia, fractures, disability after stroke (with the modified Rankin scale), cardiovascular events, hospitalisation for heart failure, hospitalisation for sepsis, and mortality.

\section{Excluded studies}

Additional file 1 provides the full list of reasons for exclusion of studies after full text analysis. The main reason for exclusion was that the study population did not match our age criteria for inclusion $(n=298)$.

\section{Main findings}

Twenty-eight studies provided evidence on relevant outcomes comparing DPP-4 inhibitors against an alternative (i.e. non-DPP-4) drug regimen or placebo. For each study and outcome Table 2 summarises the results for the DPP-4 inhibitor and comparison groups, provides estimated risk ratios with $95 \%$ confidence intervals, and reports any statistical comparisons from the study itself. To help interpretation, Table 2 organises the results first by Tier of outcome (Tier 1 or Tier 2), and then by form of comparison within Tier (DPP-4 inhibitors versus placebo; versus other active treatments; and as an additional treatment). Two further studies (not tabulated) compared between different DPP-4 inhibitor based-treatments: 1) insulin plus $100 \mathrm{mg}$ vildagliptin versus insulin plus $50 \mathrm{mg}$ vildagliptin dose [56]; 2) vildagliptin plus metformin versus vildagliptin plus 2 oral antidiabetic agents (metformin, sulfonylureas and/or thiazolidinediones) [57]. Quality of studies is also reported in Table 2.

\section{Comparisons between DPP-4 inhibitors and other drug regimens or placebo}

Nineteen studies provided evidence on Tier 1 outcomes. Studies varied in what they classified as adverse events, and as serious, severe, or significant adverse events. Hypoglycaemia was defined by 3 studies as hypoglycaemic symptoms confirmed by self-monitoring of blood glucose $<3.1 \mathrm{mmol} / \mathrm{l}[29,40, \quad 52]$; another study defined symptomatic hypoglycaemia as an episode with clinical symptoms without regard to glucose level, asymptomatic hypoglycaemia was defined as an episode of glucose level $\leq 70 \mathrm{mg} / \mathrm{dL}$ without symptoms [37]; another study defined confirmed hypoglycaemia as a symptomatic or asymptomatic event with plasma glucose $<3.0 \mathrm{mmol} / \mathrm{l}$ without requiring external assistance, severe hypoglycaemia was defined as symptomatic event requiring external assistance without regard to plasma glucose level [39]; the other 3 studies did not provide a definition of hypoglycaemia. In 10 studies [37, 39, 40, 46, 49, 50, 53-55, 59] hypoglycaemia was considerably less frequent in older people treated with DPP-4 inhibitors than in older people on other treatments, on placebo, or when used as an additional medication.

Eleven studies [29-32, 37-40,52, 53, 59] reported on adverse events other than hypoglycaemia. All showed only small, non-significant, differences. Two studies reported on fractures, one an RCT comparing saxagliptin to placebo [43] and the other a retrospective cohort study comparing DPP-4 inhibitors to other non-insulin anti-diabetic drugs [45]; both finding no significant difference. A nested case-control study reported that hospitalisation for sepsis was not significantly different between cases and controls using DPP-4 inhibitors [36].

Thirteen studies considered Tier 2 endpoints. The results for these generally more impactful outcomes were much more variable. The meta-analysis by Johansen et al. (2012) found that major cardiovascular events (fatal or non-fatal myocardial infarction or stroke, or hospitalisation for unstable angina pectoris) were significantly reduced by around $70 \%$ with linagliptin compared to comparators (mostly patients on placebo, but including a minority on anti-diabetic drugs) [58]. However, the large-scale RCTs [12, 13, 42, 44] reported no significant difference between DPP4 inhibitors (sitagliptin, saxagliptin, and alogliptin, respectively) and placebo using a similar endpoint; while one of them found a statistically significant $47 \%$ higher risk of hospitalisation for heart failure in the saxagliptin group [41]. Four observational studies reported no significant differences between DPP-4 inhibitors and other active treatments for various cardiovascular outcomes such as myocardial infarction, heart failure, ischaemic stroke, and hospitalisation for heart failure [33, 34, 47, 48], although in one study all-cause mortality was significantly lower in users of DPP-4 inhibitors than in controls [48]. One retrospective observational study reported significantly lower percentages of cardiovascular 
Table 2 Summary of study findings

\begin{tabular}{|c|c|c|c|c|c|c|}
\hline $\begin{array}{l}\text { Authors and } \\
\text { publication year }\end{array}$ & Outcomes & $\begin{array}{l}\text { DPP-4 inhibitor } \\
\text { cases } / \mathrm{n}^{\mathrm{a}}(\%)\end{array}$ & $\begin{array}{l}\text { Comparator } \\
\text { cases } / \mathrm{n}^{\mathrm{a}}(\%)\end{array}$ & $\begin{array}{l}\text { Risk ratio } \\
(95 \% \mathrm{Cl})\end{array}$ & $\begin{array}{l}\text { Reported Statistical } \\
\text { comparison }\end{array}$ & $\begin{array}{l}\text { Result } \\
\text { favours }\end{array}$ \\
\hline \multicolumn{7}{|c|}{ Tier 1 outcomes (hypoglycaemia and adverse events), comparisons against placebo } \\
\hline \multirow{5}{*}{$\begin{array}{l}\text { Barnett et al. } 2013 \text { [31] } \\
\text { QA }^{d}=\text { moderate }\end{array}$} & SAES & Linagliptin & Placebo & & & \\
\hline & & 14/162 (8.6) & $5 / 79(6.3)$ & $\begin{array}{l}1.37(0.51 \\
3.66)\end{array}$ & NR & C \\
\hline & Severe AEs & $9 / 162(5.6)$ & $3 / 79(3.8)$ & $\begin{array}{l}1.46(0.41 \\
5.25)\end{array}$ & NR & C \\
\hline & Significant AEs & $4 / 162(2.5)$ & $0 / 79(0.0)$ & $\begin{array}{l}4.40(0.24 \\
80.8)\end{array}$ & NR & C \\
\hline & Hypoglycaemia & $37 / 162(22.8)$ & $13 / 79(16.5)$ & $\begin{array}{l}1.39(0.78 \\
2.46)\end{array}$ & NR & C \\
\hline \multirow{4}{*}{$\begin{array}{l}\text { Barzilai et al. } 2011 \text { [30] } \\
\mathrm{QA}^{d}=\text { moderate }\end{array}$} & & Sitagliptin & Placebo & & & \\
\hline & Clinical AEs & 47/102 (46.1) & 55/104 (52.9) & $\begin{array}{l}0.87(0.59 \\
1.29)\end{array}$ & $\begin{array}{l}\text { Diff in } \%=-6.8 \% \\
(-20.0,6.7)\end{array}$ & $D$ \\
\hline & Clinical SAES & $7 / 102(6.9)$ & 14/104 (13.5) & $\begin{array}{l}0.51(0.21 \\
1.26)\end{array}$ & $\begin{array}{l}\text { Diff in } \%=-6.6 \% \text {, } \\
(-15.2,1.9)\end{array}$ & $D$ \\
\hline & Hypoglycaemia & 0/102 (0.0) & 0/102 (0.0) & $\begin{array}{l}1.0(0.02 \\
49.9)\end{array}$ & NR & Neither \\
\hline SAVOR-TIMI 53 & & Saxagliptin & Placebo & & & \\
\hline $\begin{array}{l}\text { Mosenzon et al. } 2015 \text { [43] } \\
\text { Subgroup P > =75 } \\
\mathrm{QA}^{\mathrm{d}}=\text { high }\end{array}$ & Bone fracture & $57 / 1169$ & $51 / 1161$ & $\begin{array}{l}1.11(0.77 \\
1.61)\end{array}$ & $\begin{array}{l}\mathrm{HR}=1.13(0.77 \\
1.65)\end{array}$ & C \\
\hline \multirow{4}{*}{$\begin{array}{l}\text { Schweizer et al. } 2013 \text { [53] } \\
\text { QA }^{d}=\text { low }\end{array}$} & & Vildagliptin & Placebo & & & \\
\hline & AEs & 29/50 (58.0) & 40/55 (72.7) & $\begin{array}{l}0.80(0.49 \\
1.29)\end{array}$ & $N R$ & $D$ \\
\hline & SAES & $7 / 50(14.0)$ & $9 / 55(16.4)$ & $\begin{array}{l}0.86(0.32, \\
2.30)\end{array}$ & NR & $D$ \\
\hline & Hypoglycaemia & $\begin{array}{l}0.49 \text { events per } \\
\text { patient-year }\end{array}$ & $\begin{array}{l}0.96 \text { events per } \\
\text { patient-year }\end{array}$ & $\begin{array}{l}0.53(0.26 \\
1.08)\end{array}$ & $p=0.970$ & $D$ \\
\hline \multirow[t]{3}{*}{$\begin{array}{l}\text { Shih et al. } 2015 \text { [36] } \\
\mathrm{QA}^{\mathrm{d}}=\text { low }\end{array}$} & $\begin{array}{l}\text { Hospitalisation } \\
\text { for sepsis: }\end{array}$ & $\begin{array}{l}\text { DPP-4 inhibitor } \\
\text { use by casese }\end{array}$ & $\begin{array}{l}\text { DPP-4 inhibitor } \\
\text { use by controls }\end{array}$ & & & \\
\hline & $\begin{array}{l}\text { Current DPP-4 } \\
\text { users only }\end{array}$ & $1148 / 43015(2.7)$ & $1152 / 43015(2.7)$ & $\begin{array}{l}1.01(0.93 \\
1.09)\end{array}$ & $\begin{array}{l}\mathrm{OR}=0.97 \\
(0.89,1.07)\end{array}$ & $D$ \\
\hline & $\begin{array}{l}\text { Used any time } \\
\text { in past year }\end{array}$ & $3523 / 43015$ (8.2) & $3276 / 43015$ (7.6) & $\begin{array}{l}1.09 \\
(1.03,1.14)\end{array}$ & $\begin{array}{l}\mathrm{OR}=1.01 \\
(0.95,1.06)\end{array}$ & C \\
\hline Strain et al. 2013 [52] & & Vildagliptin & Placebo & & & \\
\hline \multirow[t]{3}{*}{$\mathrm{QA}^{\mathrm{d}}=$ high } & AEs & $66 / 139(47.5)$ & 63/139 (45.3) & $\begin{array}{l}1.05(0.81 \\
1.35)\end{array}$ & NR & C \\
\hline & SAES & 8/139 (5.8) & 5/139 (3.6) & $\begin{array}{l}1.60(0.54 \\
4.77)\end{array}$ & $N R$ & $C$ \\
\hline & Hypoglycaemia & $3 / 139(2.2)$ & $1 / 139(0.7)$ & $\begin{array}{l}3.00(0.32 \\
28.5)\end{array}$ & $N R$ & C \\
\hline
\end{tabular}

Tier 1 outcomes (hypoglycaemia and adverse events), comparisons against other active treatments

$\begin{array}{ll}\text { Banerji et al. 2010 [29] } & \begin{array}{l}\text { Normal renal } \\ \text { function }\end{array} \\ \mathrm{QA}^{\text {d }}=\text { low } & \text { AEs } \\ \text { Subgroup } P \geq 65 & \text { SAEs } \\ & \begin{array}{l}\text { Mildly impaired } \\ \text { renal function }\end{array} \\ & \text { AEs }\end{array}$

Vildagliptin $+\quad$ TZD + metformin metformin

$1.09(0.76, \quad$ NR

54/144 (37.5) 29/84 (34.5)

1.56)

$2 / 144(1.4)$

$1 / 84(1.2)$

$1.17(0.11, \quad \quad N R$

12.7)

59/171 (34.5)

32/77 (41.6)

$0.83(0.59, \quad$ NR 
Table 2 Summary of study findings (Continued)

\begin{tabular}{|c|c|c|c|c|c|c|}
\hline & SAES & $5 / 171(2.9)$ & $4 / 77(5.2)$ & $\begin{array}{l}0.56(0.16 \\
2.04)\end{array}$ & $N R$ & $\mathrm{D}$ \\
\hline \multirow{2}{*}{$\begin{array}{l}\text { Ferrannini et al. } 2009 \text { [54] } \\
\mathrm{QA}^{d}=\text { low } \\
\text { Subgroup } P \geq 65\end{array}$} & \multirow{3}{*}{$\begin{array}{l}\text { Hypoglycaemic } \\
\text { events }\end{array}$} & Vildagliptin & Glimepiride & & & \\
\hline & & $6 / 351(1.7)$ & 59/361 (16.4) & $\begin{array}{l}0.1(0.05 \\
0.24)\end{array}$ & NR & D \\
\hline \multirow{5}{*}{$\begin{array}{l}\text { Hartley } 2015[37] \\
\mathrm{QA}^{\mathrm{d}}=\text { low }\end{array}$} & & Sitagliptin & Glimepiride & & & \\
\hline & AEs & 118/241 (49.0) & $115 / 236(48.7)$ & $\begin{array}{l}1.00(0.84 \\
1.21)\end{array}$ & $N R$ & Neither \\
\hline & SAES & $7 / 241(2.9)$ & $6 / 236(2.5)$ & $\begin{array}{l}1.14(0.39 \\
3.35)\end{array}$ & $N R$ & C \\
\hline & $\begin{array}{l}\text { Asymptomatic } \\
\text { hypoglycemia }\end{array}$ & 16/241 (6.6) & $35 / 236(14.8)$ & $\begin{array}{l}0.45(0.25 \\
0.79)\end{array}$ & $N R$ & D \\
\hline & $\begin{array}{l}\text { Symptomatic } \\
\text { hypoglycemia }\end{array}$ & $2 / 241(0.8)$ & $11 / 236(4.7)$ & $\begin{array}{l}0.18(0.04 \\
0.79)\end{array}$ & $p=0.009$ & $D$ \\
\hline \multirow{2}{*}{$\begin{array}{l}\text { Matthews et al. } 2010 \text { [55] } \\
\mathrm{QA}^{\mathrm{d}}=\text { low } \\
\text { Subgroup } P \geq 65\end{array}$} & & Vildagliptin & Glimepiride & & & \\
\hline & Hypoglycaemia & $8 / 392(2.1)$ & 69/397 (17.5) & $\begin{array}{l}0.12(0.06 \\
0.24)\end{array}$ & $p<0.001$ & D \\
\hline \multirow{3}{*}{$\begin{array}{l}\text { Penfornis et al. } 2012 \text { [49] } \\
\mathrm{QA}^{\mathrm{d}}=\text { low }\end{array}$} & & DPP-4 inhibitors & COAD & & & \\
\hline & Hypoglycaemia & 60/931 (6.4) & $52 / 257(20.1)$ & $\begin{array}{l}0.32(0.23 \\
0.45)\end{array}$ & $p<0.001$ & $D$ \\
\hline & $\begin{array}{l}\text { Severe } \\
\text { hypoglycaemia }\end{array}$ & $1 / 931(0.1)$ & $6 / 257(2.4)$ & $\begin{array}{l}0.05(0.01 \\
0.38)\end{array}$ & $p=0.001$ & D \\
\hline \multirow{4}{*}{$\begin{array}{l}\text { Rosenstock et al. } 2013 \text { [59] } \\
\mathrm{QA}^{\mathrm{d}}=\text { low }\end{array}$} & & Alogliptin & Glipizide & & & \\
\hline & Hypoglycaemia & $12 / 222(5.4)$ & $57 / 219(26.0)$ & $\begin{array}{l}0.21(0.11 \\
0.39)\end{array}$ & $N R$ & D \\
\hline & AES & 163/222 (73.4) & $151 / 219$ (68.9) & $\begin{array}{l}1.06(0.85 \\
1.33)\end{array}$ & NR & C \\
\hline & SAES & 16/222 (7.2) & 13/219 (5.9) & $\begin{array}{l}1.21(0.58 \\
2.52)\end{array}$ & $N R$ & C \\
\hline \multirow[t]{6}{*}{$\begin{array}{l}\text { Schernthaner et al. } 2015 \text { [59] } \\
\mathrm{QA}^{d}=\text { low }\end{array}$} & & $\begin{array}{l}\text { Saxagliptin }+ \\
\text { metformin }\end{array}$ & $\begin{array}{l}\text { Glimepiride } \\
\text { + metformin }\end{array}$ & & & \\
\hline & Hypoglycaemia & 21/359 (5.8) & $125 / 359(34.8)$ & $\begin{array}{l}0.17(0.11 \\
0.26)\end{array}$ & $N R$ & $D$ \\
\hline & $\begin{array}{l}\text { Severe } \\
\text { hypoglycaemia }\end{array}$ & $4 / 359(1.1)$ & 55/359 (15.3) & $\begin{array}{l}0.07(0.03 \\
0.20)\end{array}$ & $\begin{array}{l}\mathrm{OR}=0.06 \\
(0.02,0.17)\end{array}$ & D \\
\hline & $\begin{array}{l}\text { AEs (excluding } \\
\text { hypoglycaemia) }\end{array}$ & 213/359 (59.3) & 213/359 (59.3) & $\begin{array}{l}1.00(0.89 \\
1.13)\end{array}$ & $N R$ & Neither \\
\hline & SAES & 41/359 (11.4) & 32/359 (8.9) & $\begin{array}{l}1.28(0.83 \\
1.99)\end{array}$ & $N R$ & $C$ \\
\hline & Deaths & $1 / 359(0.3)$ & $1 / 359(0.3)$ & $\begin{array}{l}1.00(0.06 \\
15.93)\end{array}$ & $N R$ & Neither \\
\hline \multirow{5}{*}{$\begin{array}{l}\text { Schweizer et al. } 2009[40] \\
\mathrm{QA}^{\mathrm{d}}=\text { low }\end{array}$} & & Vildagliptin & Metformin & & & \\
\hline & AEs & 74/167 (44.3) & $83 / 165$ (50.3) & $\begin{array}{l}0.88(0.70 \\
1.11)\end{array}$ & $N R$ & $\mathrm{D}$ \\
\hline & SAES & $5 / 167(3.0)$ & 6/165 (3.6) & $\begin{array}{l}0.82(0.26 \\
2.65)\end{array}$ & $N R$ & D \\
\hline & $\begin{array}{l}\text { Gastrointestinal } \\
\text { AEs }\end{array}$ & 25/167 (15.0) & $41 / 165$ (24.8) & $\begin{array}{l}0.60(0.38 \\
0.94)\end{array}$ & NR & $\mathrm{D}$ \\
\hline & Hypoglycaemia & $0 / 167(0.0)$ & 2/165 (1.2) & $\begin{array}{l}0.20(0.01 \\
4.09)\end{array}$ & NR & $D$ \\
\hline $\begin{array}{l}\text { Sicras-Mainar and Navarro- } \\
\text { Artieda } 2014 \text { [50] }\end{array}$ & & $\begin{array}{l}\text { Vildagliptin }+ \\
\text { metformin }\end{array}$ & $\begin{array}{l}\text { Sulfonylureas + } \\
\text { metformin }\end{array}$ & & & \\
\hline
\end{tabular}


Table 2 Summary of study findings (Continued)

\begin{tabular}{|c|c|c|c|c|c|c|}
\hline $\mathrm{QA}^{\mathrm{d}}=$ very low & Hypoglycaemia & $47 / 270(17.4)$ & $307 / 717(42.8)$ & $\begin{array}{l}0.41(0.31 \\
0.53)\end{array}$ & $p<0.001$ & $\mathrm{D}$ \\
\hline \multirow[t]{2}{*}{$\begin{array}{l}\text { Viljoen et al. } 2013 \text { [46] } \\
\mathrm{QA}^{d}=\text { very low }\end{array}$} & & DPP-4 inhibitors & $\begin{array}{l}\text { Never treated } \\
\text { with DPP-4 }\end{array}$ & & & \\
\hline & Hypoglycaemia & $4 / 129(3.1)$ & 24/302 (7.9) & $\begin{array}{l}0.39(0.14 \\
1.10)\end{array}$ & $p=0.062$ & D \\
\hline \multirow[t]{3}{*}{$\begin{array}{l}\text { Driessen et al. } 2014 \text { [45] } \\
\mathrm{QA}^{d}=\text { low }\end{array}$} & Fractures & DPP-4 inhibitor & $\begin{array}{l}\text { Other non-insulin } \\
\text { anti-diabetic drugs }\end{array}$ & & & \\
\hline & $70-79$ years & NR & NR & & $\begin{array}{l}\mathrm{HR}=1.16 \\
(0.95,1.42)\end{array}$ & C \\
\hline & $80+$ years & NR & $N R$ & & $\begin{array}{l}H R=1.0 \\
(0.74,1.34)\end{array}$ & Neither \\
\hline
\end{tabular}

Tier 1 outcomes (hypoglycaemia and adverse events), DPP-4 inhibitors as an additional treatment

$$
\begin{aligned}
& \text { Chien et al. } 2011 \text { [32] } \\
& \mathrm{QA}^{\mathrm{d}}=\text { low }
\end{aligned}
$$

Kadowaki et al. 2014 [38]

Subgroup $P \geq 65$

$\mathrm{QA}^{\mathrm{d}}=1 \mathrm{OW}$

$\begin{array}{ll}\text { AEs } & 5 / 49(10.2) \\ \text { Hypoglycaemia } & 1 / 49(2.0) \\ & \begin{array}{l}\text { Teneligliptin + } \\ \text { glimepiride }\end{array} \\ \begin{array}{ll}\text { AEs (including } \\ \text { hypoglycaemia) }\end{array} & 0 / 27(0.0) \\ \begin{array}{l}\text { ADRs (including } \\ \text { hypoglycaemia) }\end{array} & 0 / 27(0.0)\end{array}$

Tier 2 outcomes (cardiovascular outcomes), comparisons against placebo

Johansen et al. 2012 [58]
$\mathrm{QA}^{d}=$ low

Subgroup $P \geq 65$

TECOS

Green et al. 2015 [13]

$\mathrm{QA}^{\mathrm{d}}=$ low

Subgroup $P \geq 65$

SAVOR-TIMI 53

Scirica et al. 2013 [12]

Scirica et al. 2014 [41]

Subgroup $P \geq 75$

Leiter et al. 2015 [42]

Subgroup $P \geq 65$

$\mathrm{QA}^{\mathrm{d}}=$ high

\section{Linagliptin}

$5 / 929(0.5)$

Fatal or non-fatal

Ml or stroke, or

hospitalisation for

unstable angina

pectoris

\begin{tabular}{ll} 
& \multicolumn{2}{l}{ Sitagliptin } \\
Composite CV & NR \\
outcome (first & \\
confirmed event & \\
of CV death, non \\
-fatal Ml, nonfatal \\
stroke, or \\
hospitalization for \\
unstable angina) \\
Subgroup $P \geq 75 \quad$ Saxagliptin \\
CV death, nonfatal & $117 / 1169$ (10.0) \\
Ml, or nonfatal & \\
ischemic stroke & \\
Hospitalisation for & $79 / 1169$ (6.8) \\
HF Subgroup $P \geq$ & \\
65 & \\
CV death, nonfatal & $334 / 4290$ (7.8) \\
Ml, or nonfatal & \\
ischemic stroke & \\
CV death, Ml, stroke, & $570 / 4290$ (13.3) \\
hospitalization for & \\
unstable angina, HF, &
\end{tabular}

OAD combinations

$3 / 49(6.1)$

0/49 (0.0)

$6.97)$

NR

$3.0(0.13$,

71.9)

NR

C

Placebo

+ glimepiride

1/34 (2.9)

1/34 (2.9)

$0.42(0.02$

9.87)

$0.42(0.02$

9.87)

NR

NR

Comparators $^{f}$

14/549 (2.6)

$0.21(0.08$

$0.58)$

$H R=0.28$,

(0.1-0.79)

Placebo

NR

$\mathrm{HR}=1.01$

$(0.90,1.15)$

C

D

D

\begin{tabular}{|c|c|c|}
\hline 129/1161 (11.3) & $\begin{array}{l}0.90(0.71 \\
1.14)\end{array}$ & $\begin{array}{l}H R=0.96 \\
(0.75,1.22)\end{array}$ \\
\hline $57 / 1161(4.9)$ & $\begin{array}{l}1.38(0.99 \\
1.92)\end{array}$ & $\begin{array}{l}H R=1.47 \\
(1.05,2.08)\end{array}$ \\
\hline $367 / 4271(8.6)$ & $\begin{array}{l}0.91(0.79, \\
1.04)\end{array}$ & $\begin{array}{l}\mathrm{HR}=0.92 \\
(0.79,1.06)\end{array}$ \\
\hline $593 / 4271(13.9)$ & $\begin{array}{l}0.96(0.86, \\
1.06)\end{array}$ & $\begin{array}{l}H R=0.96 \\
(0.85,1.07)\end{array}$ \\
\hline
\end{tabular}

Placebo
C

C

D 
Table 2 Summary of study findings (Continued)

\begin{tabular}{|c|c|c|c|c|c|c|}
\hline & \multicolumn{6}{|l|}{$\begin{array}{l}\text { or coronary } \\
\text { revascularization }\end{array}$} \\
\hline & Ml & $141 / 4290$ (3.3) & 170/4271(4.0) & $\begin{array}{l}0.83(0.66 \\
1.03)\end{array}$ & $\begin{array}{l}\mathrm{HR}=0.86 \\
(0.69,1.07)\end{array}$ & D \\
\hline & CV mortality & $158 / 4290(3.7)$ & 166/4271(3.9) & $\begin{array}{l}0.95(0.77 \\
1.17)\end{array}$ & $\begin{array}{l}\mathrm{HR}=0.92 \\
(0.74,1.13)\end{array}$ & $\mathrm{D}$ \\
\hline & Non-CV mortality & $98 / 4290(2.3)$ & $76 / 4271(1.8)$ & $\begin{array}{l}1.28(0.95 \\
1.73)\end{array}$ & $\begin{array}{l}\mathrm{HR}=1.22 \\
(0.92,1.63)\end{array}$ & C \\
\hline & All-cause mortality & 253/4290 (5.9) & 239/4271(5.6) & $\begin{array}{l}1.05(0.89 \\
1.25)\end{array}$ & $\begin{array}{l}\mathrm{HR}=1.01 \\
(0.86,1.20)\end{array}$ & C \\
\hline & $\begin{array}{l}\text { Nonfatal ischemic } \\
\text { stroke }\end{array}$ & $77 / 4290(1.8)$ & $68 / 4271(1.6)$ & $\begin{array}{l}1.13(0.82, \\
1.56)\end{array}$ & $\begin{array}{l}\mathrm{HR}=1.17 \\
(0.85,1.61)\end{array}$ & C \\
\hline & \multicolumn{6}{|l|}{$\begin{array}{l}\text { Hospitalisation for } \\
\text { /due to: }\end{array}$} \\
\hline & $C R$ & $210 / 4290(4.9)$ & 234/4271(5.5) & $\begin{array}{l}0.89(0.75 \\
1.07)\end{array}$ & $\begin{array}{l}\mathrm{HR}=0.87 \\
(0.73,1.05)\end{array}$ & D \\
\hline & $\mathrm{HF}$ & $180 / 4290(4.2)$ & 149/4271(3.5) & $\begin{array}{l}1.20(0.97 \\
1.49)\end{array}$ & $\begin{array}{l}\mathrm{HR}=1.25 \\
(1.01,1.56)\end{array}$ & C \\
\hline & Hypoglycaemia & $34 / 4290(0.8)$ & $25 / 4271(0.6)$ & $\begin{array}{l}1.35(0.81 \\
2.27)\end{array}$ & $\begin{array}{l}H R=1.29 \\
(0.78,2.14)\end{array}$ & C \\
\hline & Unstable angina & $38 / 4290(0.9)$ & $38 / 4271(0.9)$ & $\begin{array}{l}1.00(0.64 \\
1.56)\end{array}$ & $\begin{array}{l}H R=0.89 \\
(0.56,1.39)\end{array}$ & D \\
\hline \multirow{2}{*}{$\begin{array}{l}\text { White et al. } 2013 \text { [44] } \\
\mathrm{QA}^{d}=\text { low }\end{array}$} & & Alogliptin & Placebo & & & \\
\hline & $\begin{array}{l}\text { Death from CV } \\
\text { causes, or nonfatal } \\
\text { Ml or stroke }\end{array}$ & $141 / 934(15.1)$ & $149 / 973(15.3)$ & $\begin{array}{l}0.99(0.8, \\
1.22)\end{array}$ & $\begin{array}{l}\mathrm{HR}=0.98 \\
(0.78,1.24)\end{array}$ & D \\
\hline
\end{tabular}

Tier 2 outcomes (cardiovascular outcomes), comparisons against other active treatments

\begin{tabular}{|c|c|c|c|c|c|c|}
\hline \multirow{5}{*}{$\begin{array}{l}\text { Chang et al. } 2015 \text { [33] } \\
\text { Subgroup } P \geq 65 \\
\text { QA }^{d}=\text { low }\end{array}$} & & \multirow{2}{*}{$\begin{array}{l}\text { DPP-4 inhibitors } \\
\text { plus metformin } \\
\text { NR }\end{array}$} & \multicolumn{3}{|l|}{$\begin{array}{l}\text { Sulfonylureas } \\
\text { plus metformin }\end{array}$} & \multirow[b]{2}{*}{$\mathrm{D}$} \\
\hline & Any CV event & & \multicolumn{2}{|l|}{ NR } & $\begin{array}{l}H R=0.86 \\
(0.72,1.02)\end{array}$ & \\
\hline & $\mathrm{Ml}$ & NR & \multicolumn{2}{|l|}{ NR } & $\begin{array}{l}H R=0.86 \\
(0.44,1.70)\end{array}$ & $\mathrm{D}$ \\
\hline & $\mathrm{HF}$ & NR & \multicolumn{2}{|l|}{ NR } & $\begin{array}{l}\mathrm{HR}=1.01 \\
(0.72,1.43)\end{array}$ & C \\
\hline & Ischaemic stroke & NR & \multicolumn{2}{|l|}{ NR } & $\begin{array}{l}H R=0.83 \\
(0.68,1.02)\end{array}$ & $D$ \\
\hline \multirow{3}{*}{$\begin{array}{l}\text { Chen et al. } 2015 \text { [34] } \\
\text { Subgroup } P \geq 75 \\
\text { QA }^{d}=\text { low }\end{array}$} & & Sitagliptin & \multicolumn{3}{|l|}{ Non-sitagliptin } & \\
\hline & $\begin{array}{l}\text { Composite of } \\
\text { ischemic stroke, } \\
\text { Ml, or CV death }\end{array}$ & $59 / 486(12.1)$ & $104 / 949(11.0)$ & $\begin{array}{l}1.11(0.82 \\
1.50)\end{array}$ & $p=0.463$ & C \\
\hline & Ischemic stroke & 42/486 (8.6) & $77 / 949(8.1)$ & $\begin{array}{l}1.07(0.74 \\
1.53)\end{array}$ & $p=0.705$ & C \\
\hline \multirow[t]{5}{*}{$\begin{array}{l}\text { Giorda et al. } 2015 \text { [48] } \\
\mathrm{QA}^{\mathrm{d}}=\text { low }\end{array}$} & & $\begin{array}{l}\text { DPP- } 4 \text { inhibitor } \\
\text { use by cases }\end{array}$ & \multicolumn{3}{|l|}{$\begin{array}{l}\text { DPP-4 inhibitor } \\
\text { use by controls }\end{array}$} & \\
\hline & $\begin{array}{l}\text { Any admission } \\
\text { for HF }\end{array}$ & 256/14613 (1.8) & $2881 / 146130(2.0)$ & $\begin{array}{l}0.89(0.78 \\
1.01)\end{array}$ & $\begin{array}{l}\mathrm{OR}=1.00 \\
(0.94,1.07)\end{array}$ & Neither \\
\hline & Incident HF & $135 / 7212(1.9)$ & $\begin{array}{l}1285 / 72120 \\
(1.8)\end{array}$ & $\begin{array}{l}1.05(0.88 \\
1.25)\end{array}$ & $\begin{array}{l}\mathrm{OR}=1.01 \\
(0.92,1.11)\end{array}$ & C \\
\hline & $\begin{array}{l}\text { Re-admission } \\
\text { for HF }\end{array}$ & $37 / 1727(2.1)$ & $\begin{array}{l}338 / 17222 \\
(2.0)\end{array}$ & $\begin{array}{l}1.09(0.78 \\
1.53)\end{array}$ & $\begin{array}{l}\mathrm{OR}=1.02 \\
(0.84,1.22)\end{array}$ & C \\
\hline & $\begin{array}{l}\text { All-cause } \\
\text { mortality }\end{array}$ & $306 / 38248(0.8)$ & $6717 / 382313(1.8)$ & $\begin{array}{l}0.46(0.41 \\
0.51)\end{array}$ & $\begin{array}{l}\mathrm{OR}=0.94 \\
(0.90,0.98)\end{array}$ & $D$ \\
\hline
\end{tabular}


Table 2 Summary of study findings (Continued)

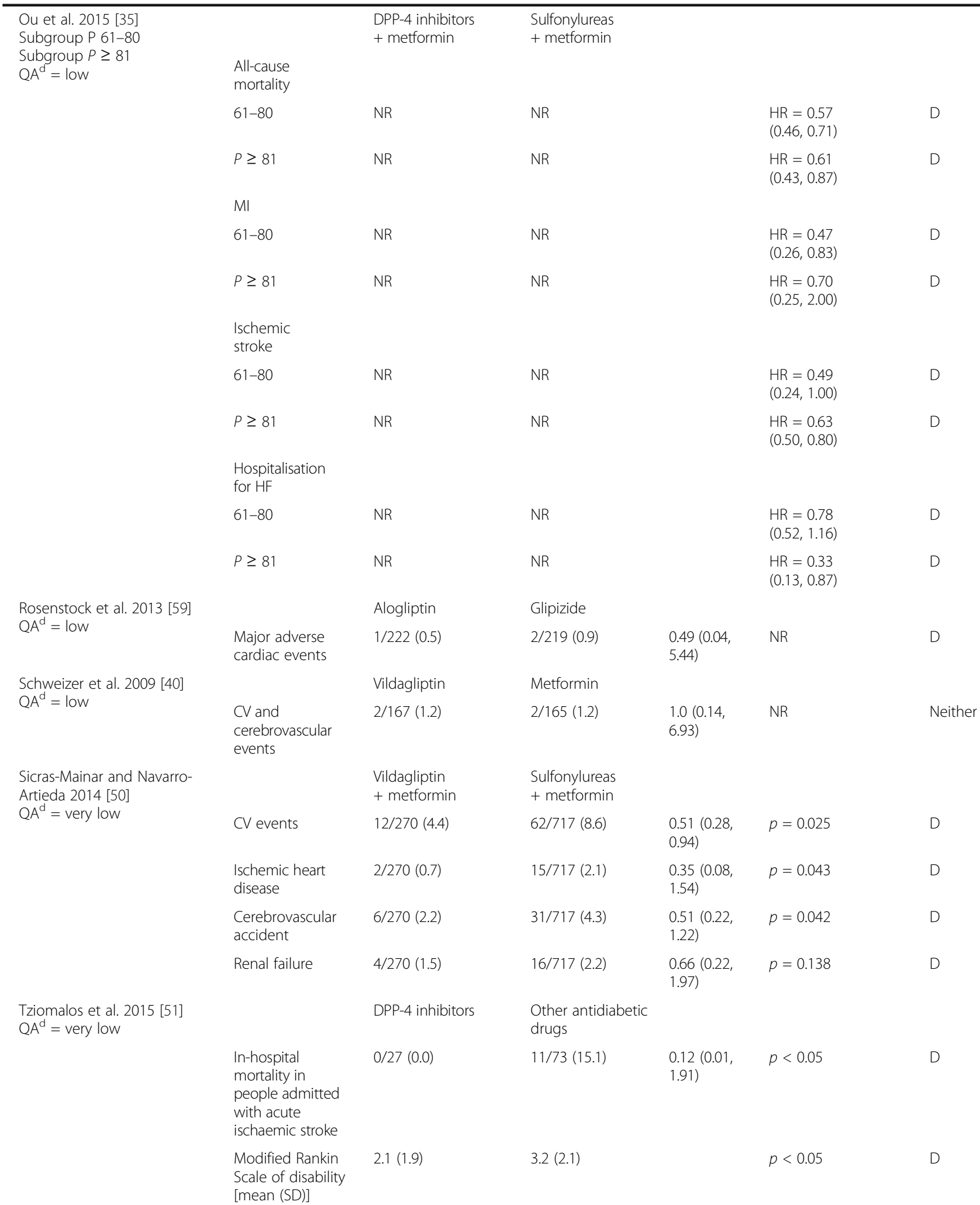


Table 2 Summary of study findings (Continued)

\begin{tabular}{|c|c|c|c|c|c|c|}
\hline \multirow[t]{2}{*}{$\begin{array}{l}\text { Yu et al. } 2015 \\
\mathrm{QA}^{\mathrm{d}}=\text { low }\end{array}$} & & $\begin{array}{l}\text { DPP-4 inhibitor } \\
\text { use by casese }\end{array}$ & $\begin{array}{l}\text { DPP- } 4 \text { inhibitor } \\
\text { use by controls }\end{array}$ & & & \\
\hline & $\begin{array}{l}\text { Hospitalisation } \\
\text { for HF }\end{array}$ & $54 / 1118(4.8)$ & $808 / 17626$ (4.6) & $\begin{array}{l}1.05(0.81 \\
1.38)\end{array}$ & $\begin{array}{l}\mathrm{OR}=0.88 \\
(0.63,1.22)\end{array}$ & D \\
\hline
\end{tabular}

events, ischemic heart disease, and cerebrovascular accident with vildagliptin plus metformin compared to sulfonylureas plus metformin [50]; but Schweizer et al. (2009) found no difference between vildagliptin and metformin in rates of cardiovascular and cerebrovascular events [40], and Rosenstock et al. (2013) observed that alogliptin and glipizide did not differ in major cardiac events, though in both of these latter randomised trials samples sizes were small and events rare [59]. A cohort study reported significantly less mortality, myocardial infarction, ischemic stroke and hospitalisation for heart failure with DPP-4 inhibitors plus metformin compared to sulfonylureas plus metformin [35]. A small observational study reported significantly lower in-hospital mortality in people admitted with acute ischemic stroke and better scores on the modified Rankin scale of disability with DPP-4 inhibitors compared to other antidiabetics [51].

We deemed study synthesis by meta-analysis inappropriate, due to high heterogeneity of treatments, outcome definitions and follow-up periods. However, to gain a global overview and aid interpretation, Table 2 indicates the treatment arm favoured on each outcome in each study, based purely on the reported direction of effect or (where missing) risk ratio point estimate and ignoring statistical significance. Under this "vote counting" method [19], for the Tier 1 outcomes 8 studies fully favoured the DPP- 4 inhibitor, 4 fully favoured the comparator, and 7 were mixed or neutral. For the Tier 2 outcomes, 7 studies favoured DPP- 4 inhibitors, 2 favoured comparators, and 4 were mixed or neutral. However, only 2 studies received high overall ratings for quality (see below); both reporting Tier 1 outcomes favouring placebo and one reporting Tier 2 outcomes with mixed results. There was no suggestion for either tier of outcomes of the pattern of results differing according to whether the comparison was a placebo, other active treatment, or DPP-4 inhibitors as an additional treatment.

\section{Comparisons between DPP-4 inhibitor-based treatments}

Both studies reported that hypoglycaemic events were similar between the groups: 1) 2.1 events per patient-year with insulin plus vildagliptin $100 \mathrm{mg}$ versus 2.3 events per patient-year with insulin plus vildagliptin $50 \mathrm{mg}$ [56]; 2) no events with vildagliptin plus metformin versus 1 event with vildagliptin plus 2 oral antidiabetic agents [57].

\section{Quality appraisal of included studies Meta-analysis}

One meta-analysis was included [58] and it met 4 out of 11 criteria of the AMSTAR tool (Table 3).

\section{Clinical trials}

Seventeen clinical trials were included and their quality appraisal is shown in Table 4. Only two studies had a low risk of bias for all seven items [12, 41-43, 52]. Four RCTs had reasonable good quality with low risk of selection, performance, and attrition bias [13, 30, 31, 37]. Most studies had low risk of attrition bias. Twelve studies were at high risk of "other bias". One study was a retrospective analysis of an open-label clinical trial and had a high risk of selection, performance and detection bias [29]. Most of the included clinical trials did not provide enough information to fully assess their risk of bias and had "unclear risk of bias" for at least four of the items.

\section{Observational studies}

Quality appraisal of the 12 observational studies is shown in Table 5. Six studies reported insufficiently on most of the CASP items to be considered of high quality $[36,46,49,50,57]$. The other six studies reported information on most of the CASP items to be considered of high quality [33-35, 45, 47, 48].

\section{Overall ratings of quality}

The single meta-analysis was rated as low quality overall because this investigation did not assess the scientific quality of its included studies. The majority of individual studies were rated low or very low overall quality, and only two received a high overall quality rating.

\section{Involvement of pharmaceutical companies in studies}

Twenty-two studies were funded by pharmaceutical companies and authored or co-authored by employees of the sponsor (22 out of 30, 73\%). In the remaining eight studies, one study gave no information about funding although 


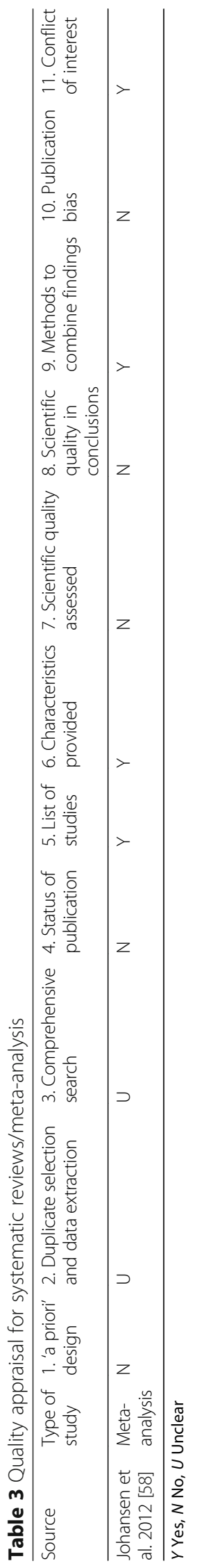


Table 4 Quality appraisal for intervention studies

\begin{tabular}{|c|c|c|c|c|c|c|c|c|}
\hline \multirow[t]{2}{*}{ Source } & \multirow[t]{2}{*}{ Type of study } & \multicolumn{2}{|c|}{ Selection bias } & \multirow{2}{*}{$\begin{array}{l}\text { Performance } \\
\text { bias } \\
\text { 3. Blinding of } \\
\text { participants } \\
\text { and personnel }\end{array}$} & \multirow{2}{*}{$\begin{array}{l}\text { Detection } \\
\text { bias } \\
\text { 4. Blinding } \\
\text { of outcome } \\
\text { assessment }\end{array}$} & \multirow{2}{*}{$\begin{array}{l}\text { Attrition bias } \\
\text { 5. Incomplete } \\
\text { outcome data }\end{array}$} & \multirow{2}{*}{$\begin{array}{l}\text { Reporting } \\
\text { bias } \\
\text { 6. Selective } \\
\text { reporting }\end{array}$} & \multirow[b]{2}{*}{$\begin{array}{l}\text { 7. Other } \\
\text { bias }\end{array}$} \\
\hline & & $\begin{array}{l}\text { 1. Random } \\
\text { sequence } \\
\text { generation }\end{array}$ & $\begin{array}{l}\text { 2. Allocation } \\
\text { concealment }\end{array}$ & & & & & \\
\hline $\begin{array}{l}\text { Banerji et al. } 2010 \\
\text { [29] }\end{array}$ & $\begin{array}{l}\text { Retrospective analysis of the } \\
\text { GALIANT study which is a } \\
\text { multicenter, randomised, } \\
\text { open-label study }\end{array}$ & $L R$ & $\mathrm{HR}$ & $\mathrm{HR}$ & $\mathrm{HR}$ & $L R$ & UR & $\mathrm{HR}$ \\
\hline $\begin{array}{l}\text { Barnett et al. } 2013 \\
\text { [31] }\end{array}$ & $\begin{array}{l}\text { Randomised, double-blind, } \\
\text { placebo-controlled trial }\end{array}$ & $L R$ & $L R$ & $L R$ & UR & $L R$ & $L R$ & UR \\
\hline $\begin{array}{l}\text { Barzilai et al. } 2011 \\
\text { [30] }\end{array}$ & $\begin{array}{l}\text { Randomised, double-blind, } \\
\text { placebo-controlled trial }\end{array}$ & $L R$ & $L R$ & $L R$ & UR & $L R$ & UR & UR \\
\hline Chien et al. 2011 [32] & $\begin{array}{l}\text { Randomised, open-labelled, } \\
\text { parallel-group study }\end{array}$ & UR & UR & UR & $\mathrm{HR}$ & UR & UR & $\mathrm{HR}$ \\
\hline $\begin{array}{l}\text { Ferrannini et al. } 2009 \\
\text { [54] }\end{array}$ & $\begin{array}{l}\text { Multicentre, randomised, } \\
\text { double-blind, active- } \\
\text { controlled study }\end{array}$ & UR & UR & UR & UR & $L R$ & UR & $H R$ \\
\hline $\begin{array}{l}\text { Fonseca et al. } 2008 \\
\text { [56] }\end{array}$ & $\begin{array}{l}\text { Multicentre, double-blind, } \\
\text { parallel-group, randomised } \\
\text { study }\end{array}$ & UR & UR & UR & UR & $L R$ & UR & $H R$ \\
\hline Green et al. 2015 [13] & $\begin{array}{l}\text { Randomised, double-blind, } \\
\text { placebo-controlled study }\end{array}$ & $L R$ & $L R$ & $L R$ & $L R$ & $L R$ & $L R$ & $H R$ \\
\hline $\begin{array}{l}\text { Hartley et al. } 2015 \\
\text { [37] }\end{array}$ & $\begin{array}{l}\text { Randomised, parallel-group, } \\
\text { multinational, non-inferiority } \\
\text { clinical trial with an active } \\
\text { controlled, double-blind } \\
\text { treatment period }\end{array}$ & $L R$ & $L R$ & LR & UR & $L R$ & $L R$ & $\mathrm{HR}$ \\
\hline $\begin{array}{l}\text { Kadowaki et al. } 2014 \\
\text { [38] }\end{array}$ & $\begin{array}{l}\text { Randomised, double-blind, } \\
\text { placebo-controlled study }\end{array}$ & UR & UR & $L R$ & UR & $L R$ & UR & $H R$ \\
\hline $\begin{array}{l}\text { Matthews et al. } 2010 \\
\text { [55] }\end{array}$ & $\begin{array}{l}\text { Multicentre, randomised, } \\
\text { double-blind, double- } \\
\text { dummy, active-controlled } \\
\text { study }\end{array}$ & UR & UR & $L R$ & UR & $L R$ & UR & $H R$ \\
\hline $\begin{array}{l}\text { Rosenstock et al. } 2013 \\
\text { [59] }\end{array}$ & $\begin{array}{l}\text { Multicentre, randomised, } \\
\text { double-blind, active } \\
\text { controlled study }\end{array}$ & UR & UR & $L R$ & UR & $L R$ & UR & $H R$ \\
\hline $\begin{array}{l}\text { Schernthaner et al. } \\
2015 \text { [39] }\end{array}$ & $\begin{array}{l}\text { Multinational, randomised, } \\
\text { double-blind, phase IIIb/IV } \\
\text { study }\end{array}$ & LR & UR & UR & UR & $H R$ & UR & $H R$ \\
\hline $\begin{array}{l}\text { Schweizer et al. } 2009 \\
\text { [40] }\end{array}$ & $\begin{array}{l}\text { Double-blind, randomised, } \\
\text { multicentre, active-controlled, } \\
\text { parallel-group study }\end{array}$ & UR & UR & UR & UR & $L R$ & UR & $H R$ \\
\hline $\begin{array}{l}\text { Schweizer et al. } 2013 \\
\text { [53] }\end{array}$ & $\begin{array}{l}\text { Post-hoc sub-analysis of a } \\
\text { multi-centre, randomised, } \\
\text { double-blind, parallel-group }\end{array}$ & UR & UR & UR & UR & UR & UR & UR \\
\hline $\begin{array}{l}\text { Scirica et al. } 2013 \text { [12] } \\
\text { Scirica et al. } 2014 \text { [41] } \\
\text { Leiter et al. } 2015 \text { [42] } \\
\text { Mosenzon et al. 2015 } \\
\text { [43] }\end{array}$ & $\begin{array}{l}\text { Multicentre, randomised, } \\
\text { double-blind, placebo- } \\
\text { controlled trial }\end{array}$ & $L R$ & $L R$ & $L R$ & $L R$ & $L R$ & $L R$ & $L R$ \\
\hline Strain et al. 2013 [52] & $\begin{array}{l}\text { Multinational, double-blind, } \\
\text { randomised, placebo- } \\
\text { controlled }\end{array}$ & $L R$ & $L R$ & $L R$ & $L R$ & $L R$ & $L R$ & $L R$ \\
\hline White et al. 2013 [44] & $\begin{array}{l}\text { Multicentre, randomised, } \\
\text { double-blind placebo- } \\
\text { controlled trial }\end{array}$ & $L R$ & UR & $L R$ & UR & $L R$ & $L R$ & $H R$ \\
\hline
\end{tabular}

$L R$ Low risk of bias, $H R$ High risk of bias, UR Unclear risk of bias 


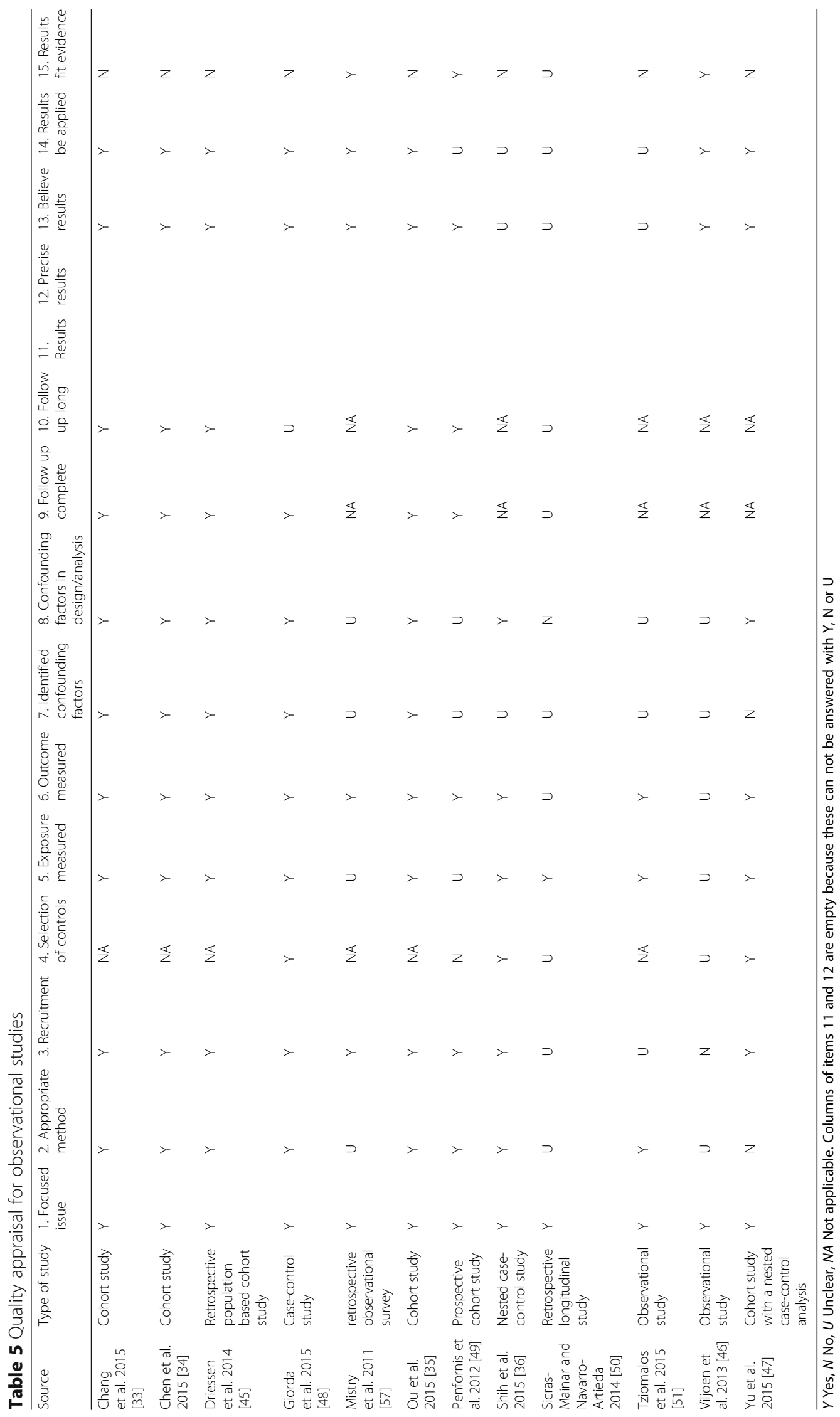


authors stated globally they had no conflict of interest [32]; two studies reported no funding and no conflict of interest [33, 51]; two studies reported funding outside pharmaceuticals and no conflict of interest $[36,48]$; two studies reported funding outside pharmaceuticals and conflict of interest from some of the authors receiving fees from pharmaceuticals [34, 47]; one study reported no funding but one author declared receiving fees from pharmaceuticals [35]. The 30 included studies were authored by 219 authors: $29 \%$ (63 out of 219) were employees of the pharmaceutical sponsor, and an additional $27 \%$ declared conflicts of interest ( 60 out of 219 , often consulting fees by the sponsor). Sixty-one authors of 11 publications (61 out of 219, 28\%) declared no conflicts of interest. Support by professional medical writers was given in at least 8 publications (8 out of $30,27 \%$ ).

\section{Additional references of interest for the development of recommendations}

We found four additional references that were taken into consideration for the development of the recommendation: 1 meta-analysis, 1 pooled analysis, 1 observational study, and a report from the FDA. These are shown in Additional file 4: Table S2. These references did not meet our age or study design criteria for inclusion. They were counted as being relevant to recommendations principally because they provided information about clinically relevant endpoints not adequately addressed by the 30 included studies, albeit for younger populations.

One of the additional references reported that there were no statistically significant differences between vildagliptin compared to other anti-diabetic treatments or placebo for long-term outcomes including acute coronary syndrome, transient ischaemic attack, stroke, myocardial infarction, cardiovascular and cerebrovascular death [60]. Two of the additional references reported an increase in the risk of hospitalisations for heart failure and an increase in heart failure outcomes in people under DDP-4 inhibitors compared to people under other anti-diabetic treatments or placebo $[61,62]$. These studies concur with that of Scirica et al. (2013) [12] for patients age 75 and over, included in the present SR.
Furthermore, the U.S. Food and Drug Administration (FDA) and the European Medicines Agency (EMA) reported that there are still some uncertainties with respect to long-term pancreatic safety with DPP-4 inhibitors and evaluation of these outcomes is ongoing [63]. Although the currently available data are reassuring, pancreatitis will continue to be considered a risk associated with these drugs until more data are available. These additional references suggest that certain risks like heart failure and related hospitalisation, and pancreatitis, may be increased with the use of DPP-4 inhibitors compared to other antidiabetic treatments, independently of age group.

\section{Recommendations}

Recommendations were developed following a standardized schema and reflecting the strength and the quality of the evidence. Two meetings were held by GS (researcher and clinician), YVM (researcher) and ARG (researcher and geriatrician), with AS participating in one of these as a senior clinician and researcher. Subsequent to these meetings we agreed a recommendation which was later confirmed with IK and MMV for its inclusion in the Comprehensive Medication Review (CMR) tool developed within the PRIMA-eDS project.

From the results of our SR and the additional references of interest we developed one recommendation in relation to DPP-4 inhibitors use in older people with type 2 diabetes. The recommendation is that the clinician should consider discontinuing gliptins where the patient has $\mathrm{HbA1c}<8.5 \%$, principally because of the sparse and inconsistent evidence for clinically relevant benefits, but taking the patient's symptoms into account (Table 6). The recommendation was considered as a weak recommendation. The quality was downgraded from high to moderate for indirectness.

We considered glycaemic control in the recommendation although it was not one of our study endpoints. The aim was that clinicians would focus on those patients who may benefit more from the recommendation as they could be already having acceptable glycaemic control. In older people, rigid glycaemic control $(<\mathrm{HbA} 1 \mathrm{c} 8.0 \%)$ has been found to be associated with a higher risk of hypoglycaemia and undesirable long-term outcomes like increased

Table 6 Recommendation for DPP-4 inhibitors in older people with type 2 diabetes

\begin{tabular}{|c|c|c|}
\hline Recommendation & Strength of the recommendation & Quality of the evidence \\
\hline $\begin{array}{l}\text { The patient is taking DPP- } 4 \text { inhibitors and } \mathrm{HbA1c} \\
\text { is }<8.5 \% \text { ( } 70 \mathrm{mmol} / \mathrm{mol} \text { ). Please reconsider the use } \\
\text { of gliptins for the management of type } 2 \text { diabetes } \\
\text { in older adults because of scarce data on clinically } \\
\text { relevant benefits of their use. Please take the } \\
\text { patient's symptoms into consideration. }\end{array}$ & $\begin{array}{l}\text { Weak } \\
\text { Reason: No trial data supporting long-term } \\
\text { clinically-relevant benefits in older people. One } \\
\text { RCT pointing at possible adverse long-term } \\
\text { effects independently from age. }\end{array}$ & $\begin{array}{l}\text { The evidence was graded low quality. It was } \\
\text { considered to downgrade the quality of the } \\
\text { evidence to low quality because there were study } \\
\text { limitations ( } 1 \text { observational study and a pooled } \\
\text { analysis), indirectness (most of the studies did not } \\
\text { report data in older people, apart from the pooled } \\
\text { analysis), inconsistency (different types of DPP-4 } \\
\text { inhibitors evaluated), and lack of data of long-term } \\
\text { benefits under DPP-4 inhibitors in older people. }\end{array}$ \\
\hline
\end{tabular}


mortality [64]. The target population in the PRIMA-eDS trial were people 75 years or older with at least eight prescribed medications reflecting a high comorbidity burden. An $\mathrm{HbA1c}<8.5 \%$ has been recommended in guidelines as a target goal in older people who have comorbidities, poor health, dementia, frailty or limited life expectancy $[8,65-$ 67]. As a general rule, PRIMA-eDS recommends clinicians to take symptoms into consideration as well as the individual participant characteristics such as frailty level and comorbidities.

\section{Discussion}

Thirty studies reported in 33 publications (one MA, 17 interventional studies and 12 observational studies) were identified which evaluated the use of gliptins for the management of type 2 diabetes in older people and reported on clinically relevant outcomes. While the majority of the studies reported participant data on comorbidities, only one presented data on frailty status. In terms of outcomes, most of the included studies reported on adverse events and hypoglycaemia. Fourteen studies reported on cardiovascular events (such as heart failure, myocardial infarction, and stroke), hospitalisation for heart failure, functional status, cardiovascular mortality, and all-cause mortality either as individual outcomes or combined into a composite outcome. None of these studies evaluated allcause hospitalisation, quality of life or cognitive status.

In general, studies of DPP-4 inhibitors have shown similar or better safety than placebo and other antidiabetic drugs in older adults with type 2 diabetes, but these safety data are mainly based on short-term outcomes like hypoglycaemic events and acute adverse events. The evidence for longer-term health or qualityof-life benefits is more limited and quite inconsistent, with some studies showing benefits and others increased risks, particularly when the evidence from younger age groups is factored in. In addition, only six studies had reasonably good quality and the results from these provided very little evidence for a benefit in older people from treatment with DPP-4 inhibitors.

DPP-4 inhibitors have been recommended as second line drugs for the management of type 2 diabetes in older adults by several expert groups [68] because of their lower risk of hypoglycaemia. Hypoglycaemia may be very relevant in older people, especially if it is symptomatic and has consequences such as falls. Unfortunately, information on the consequences of the hypoglycaemic events was not clearly reported in most studies. However, the majority of studies included patients with a mean $\mathrm{HbA} 1 \mathrm{c} \leq 8 \%$ at baseline. According to current guidelines, for older patients with these HbA1c levels further treatment may not be recommended, especially in those with functional impairment $[8,10,69]$. Rigid glycaemic control beyond an HbA1c of $8 \%$ achieved by antidiabetic drugs may be associated with a higher risk of hypoglycaemia and undesirable long-term outcome like increased mortality [64]. At present, it is unclear if the decreased risk for hypoglycaemia seen with the use of gliptins would also be seen in populations with less tight glycaemic control. In terms of effectiveness, we did not use glycaemic control as a clinically relevant endpoint. Glycaemic control has often been regarded as a surrogate endpoint without evidence for a direct relationship to longer term outcomes [70]. However, our omission of evidence for good glycaemic control with less adverse events might have introduced a limitation to our systematic review.

Unfortunately, most studies included in this publication did not provide any information on the frailty level and cognitive status of their participants. Data on some comorbidities and the use of some concomitant drugs were provided but the number of drugs used and concomitant diseases were rarely reported. This limits the interpretation of the results and their applicability to the heterogeneous older population.

Furthermore, with the exception of five studies [32, 33, $36,48,51]$, the rest of the included studies on DPP-4 inhibitors in older people were sponsored by pharmaceutical companies, authored or co-authored by company employees, or included authors working closely with the pharmaceutical sponsor and receiving consultancy or advisory fees $(60 / 219,28 \%)$. A close affiliation between pharmaceutical companies and researchers appears to be a general problem in diabetes research [71] and raises concerns about the independence and integrity of the evidence base for the treatment of diabetes.

Five studies were from Taiwan including a randomised trial [32] and four observational studies [33-36]. All of these observational studies used the same database from the Taiwan National Health Insurance. Although it seems that populations are different in each of these studies, three of them were on DPP-4 inhibitors [33, 35, 36]. It might have been possible that some of the samples included similar populations.

We conducted this SR following an adaption of the standard methodology recommended by the Cochrane collaboration and the PRISMA statement. The searches were conducted in six biomedical literature databases. We developed a stepwise search as part of the methodology for this systematic review and included existing systematic reviews and meta-analyses, as well as individual studies. However, this methodology has not been independently validated. Search $3 \mathrm{~B}$ was limited to publications since 2005 [21]. Although it is conceivable that some pre-2005 studies were missed in this process, we believe that earlier relevant studies were captured during search $3 \mathrm{~A}$, checking of references lists, or snowballing. Furthermore, the first DPP-4 inhibitor (i.e. 
sitagliptin) was only approved by the FDA in 2006 which gives us confidence that we did not miss relevant studies [72]. Many patients are prescribed combinations of antidiabetic medications and we have not attempted to modulate our recommendation according to the particular treatments being used alongside DPP-4 inhibitors or when these are the sole treatment. This is an important but complex issue that could not be addressed within the objectives of this systematic review, and when re-considering the use of DPP-4 inhibitors with an individual patient, the clinician must take into account any additional treatments the patient may be receiving for their diabetes.

The recommendation derived from the results of this SR aims at increasing awareness in clinicians about the evidence (and lack of evidence) with regard to the use of DPP4 inhibitors for the management of type 2 diabetes in older adults. Decisions on the prescription or de-prescription of DPP-4 inhibitors should be made taking the symptoms and individual characteristics of each patient into account, including any other antidiabetic medications the patient may be taking, and involving the older person themselves in the decision-making process [73]. HbA1c levels should also be taken into consideration as current guidelines recommend no further treatment in older people with functional impairment and HbA1c <8\% [8, 10,69]. We developed the recommendation based on the results of this SR and the four additional references (studies without age or study design criteria to be included) which provided information about clinically relevant endpoints not adequately addressed by the 30 included studies. It should be noted that the included studies in this SR only provided evidence of a suspected effect on hospitalisations for heart failure with saxagliptin [41, 42]. However, additional references suggest that certain risks like heart failure and related hospitalisation, and pancreatitis, may be increased with the use of DPP-4 inhibitors compared to other anti-diabetic treatments, independently of age group [60-63].

The results of this SR show that further research is needed on the clinically relevant short and long-term risks and benefits of the use of DPP-4 inhibitors for the management of type 2 diabetes in older adults. Older adults living in different settings including care homes, with comorbidities, polypharmacy, cognitive and functional limitations should be represented in the studies.

\section{Conclusions}

Evidence for beneficial clinically relevant outcomes regarding the usage of DPP-4 inhibitors in older people with type 2 diabetes is ambiguous at best. DPP-4 inhibitors appear to be safer compared to other anti-diabetic medications to treat older people with type 2 diabetes. However, these safety data are based only on short-term surrogate outcomes and standard HbA1c control targets, and the characteristics of the studied older people in terms of frailty and medical complexity are not described. In addition, there is a lack of studies independent of pharmaceutical company sponsorship. Independently from age, an increased risk of heart failure outcomes in adults under DDP-4 inhibitors has been reported [74]. Therefore, at present, DPP-4 inhibitors should be prescribed with caution in older patients with type 2 diabetes, especially if HbA1c is already in the therapeutic range of $<8.5 \%$ recommended by experts for frail older people (from expert-based recommendations).

\section{Additional files}

Additional file 1: Excluded studies. List of excluded studies after full-text check with reasons for exclusion. (XLSX $47 \mathrm{~kb}$ )

Additional file 2: Search strategy. Full search terms for each search (searches 1, 2 and 3B). (DOCX $185 \mathrm{~kb}$ )

Additional file 3: Participant characteristics. Characteristics of the participants in the included studies. (DOCX $60 \mathrm{~kb}$ )

Additional file 4: Additional evidence for recommendation. Additional evidence for recommendation. (DOCX $15 \mathrm{~kb}$ )

\section{Abbreviations}

AMSTAR: A Measurement Tool to Assess Systematic Reviews; CASP: Critical Appraisal Skills Programme; CMR: Comprehensive Medication Review; DARE: Database of Abstracts or Reviews of Effects; GRADE: Grading of Recommendations Assessment, Development and Evaluation; HTA: Health Technology Assessment; IPA: International Pharmaceutical Abstracts; PICOS: Population, intervention, comparison, outcomes and study design; PRIMA-eDS: Polypharmacy in chronic diseases: Reduction of Inappropriate Medication and Adverse drug events in elderly populations by electronic Decision Support; PRISMA: Preferred Reporting Items for Systematic Reviews and Meta-Analyses; RCTs: Randomised controlled trials; SR: Systematic review

\section{Acknowledgements}

We would like to thank Adrine Woodham at the University of Manchester for her support conducting the database searches.

\section{Funding}

The PRIMA-eDS study was supported by a grant from the European Commission within the 7th Framework Programme (Grant No. 305388-2). The work of YM was also supported by a grant from the NIHR Greater Manchester Primary Care Patient Safety Translational Research Centre.

Publication of this article was funded by the UK National Institute for Health Research School for Primary Care Research, University of Manchester.

\section{Availability of data and materials \\ The data supporting the conclusions of this article is included within the article (and its additional files).}

\section{About this supplement}

This article has been published as part of BMC Geriatrics Volume 17 Supplement 1, 2017: The Evidence Base of Frequently prescribed drugs in older Patients: A series of systematic reviews as a basis for recommendations in the PRIMA-eDStool to reduce inappropriate polypharmacy. The full contents of the supplement are available online at https://bmcgeriatr.biomedcentral.com/articles/supplements/ volume-17-supplement-1.

\section{Authors' contributions}

AS, REED and YVM conceptualised the study. GS, AV, YMV and REED selected the included studies. GS, YMV and REED extracted data and conducted quality appraisal. GS, YVM and ARG prepared the recommendation to be discussed with AS, IK, and MMV. GS, YVM and REED drafted the manuscript, 
supported by DR and AS. All the authors critically reviewed the drafts and approved the final manuscript.

\section{Ethics approval and consent to participate}

Not applicable.

\section{Consent for publication}

Not applicable.

\section{Competing interests}

The authors declare that they have no competing interests.

\section{Publisher's Note}

Springer Nature remains neutral with regard to jurisdictional claims in published maps and institutional affiliations.

\begin{abstract}
Author details
'Drug Commission of the German Medical Association, Berlin, Germany. ${ }^{2} \mathrm{NIHR}$ School for Primary Care Research, Manchester Academic Health Science Centre, University of Manchester, Manchester, England. ${ }^{3}$ Family Medicine Unit, Faculty of Medicine, University of Colombo, Colombo, Sri Lanka. ${ }^{4}$ Institute of General Practice and Family Medicine, Witten/Herdecke University, Witten, Germany. ${ }^{5}$ Department of Geriatrics, University Hospital Parc de Salut Mar, Barcelona, Spain. ${ }^{6}$ South Tyrolean Academy of General Practice, Bolzano, Italy. ${ }^{7}$ Duodecim Medical Publications Ltd, Helsinki, Finland.
\end{abstract}

\section{Published: 16 October 2017}

\section{References}

1. International Diabetes Federation: IDF Diabetes Atlas. In., 7th edn. Brussels, Belgium: International Diabetes Federation; 2015. http://www.diabetesatlas. org/. Accessed 20 Aug 2017.

2. World Health Organization. Diabetes. Fact sheet N³12. http://who.int/ mediacentre/factsheets/fs312/en/. Accessed 20 Aug 2017.

3. World Health Organization. Prevention of Diabetes Mellitus. Technical Report Series No. 844; 1994.

4. Gurwitz JH, Field TS, Harrold LR, Rothschild J, Debellis K, Seger AC, Cadoret C, Fish LS, Garber L, Kelleher M, et al. Incidence and preventability of adverse drug events among older persons in the ambulatory setting. JAMA. 2003:289(9):1107-16.

5. Kirkman MS, Briscoe VJ, Clark N, Florez H, Haas LB, Halter JB, Huang ES, Korytkowski MT, Munshi MN, Odegard PS, et al. Diabetes in older adults. Diabetes Care. 2012:35(12):2650-64.

6. Thornberry NA, Gallwitz B. Mechanism of action of inhibitors of dipeptidylpeptidase-4 (DPP-4). Best Pract Res Clin Endocrinol Metab. 2009;23(4):479-86.

7. National Institute for Health and Care Excellence: Type 2 diabetes in adults: management. Clinical Guideline Update (NG28). London; 2015.

8. American Diabetes Association. 10. Older Adults. Diabetes Care. 2016; 39(Supplement 1):S81-5.

9. Inzucchi SE, Bergenstal RM, Buse JB, Diamant M, Ferrannini E, Nauck M, Peters AL, Tsapas A, Wender R, Matthews DR, et al. Management of hyperglycemia in type 2 diabetes: a patient-centered approach: position statement of the American Diabetes Association (ADA) and the European Association for the Study of diabetes (EASD). Diabetes Care. 2012;35(6):1364-79.

10. Bundesärztekammer (BÄK), Kassenärztliche Bundesvereinigung (KBV), Arbeitsgemeinschaft der Wissenschaftlichen Medizinischen Fachgesellschaften (AWMF). Nationale VersorgungsLeitlinie Therapie des Typ-2-Diabetes - Langfassung, 1. Auflage. Version 4. 2013, zuletzt geändert: November 2014. Available from: http://www.leitlinien.de/nvl/diabetes/ therapie. Accessed 20 Aug 2017.

11. Karagiannis T, Paschos P, Paletas K, Matthews DR, Tsapas A. Dipeptidyl peptidase-4 inhibitors for treatment of type 2 diabetes mellitus in the clinical setting: systematic review and meta-analysis. BMJ. 2012;344:e1369.

12. Scirica BM, Bhatt DL, Braunwald E, Steg PG, Davidson J, Hirshberg B, Ohman P, Frederich R, Wiviott SD, Hoffman EB, et al. Saxagliptin and cardiovascular outcomes in patients with type 2 diabetes mellitus. N Engl J Med. 2013;369(14):1317-26.

13. Green JB, Bethel MA, Armstrong PW, Buse JB, Engel SS, Garg J, Josse R, Kaufman KD, Koglin J, Korn S, et al. Effect of Sitagliptin on cardiovascular outcomes in type 2 diabetes. N Engl J Med. 2015;373(3):232-42.
14. Zannad F, Cannon CP, Cushman WC, Bakris GL, Menon V, Perez AT, Fleck PR, Mehta CR, Kupfer S, Wilson C, et al. Heart failure and mortality outcomes in patients with type 2 diabetes taking alogliptin versus placebo in EXAMINE: a multicentre, randomised, double-blind trial. Lancet. 2015:385(9982):2067-76.

15. Crome P, Lally F, Cherubini A, Oristrell J, Beswick AD, Clarfield AM, Hertogh C, Lesauskaite V, Prada Gl, Szczerbinska K, et al. Exclusion of older people from clinical trials: professional views from nine European countries participating in the PREDICT study. Drugs Aging. 2011;28(8):667-77.

16. Van Spall HG, Toren A, Kiss A, Fowler RA. Eligibility criteria of randomized controlled trials published in high-impact general medical journals: a systematic sampling review. JAMA. 2007;297(11):1233-40.

17. Boyd CM, Darer J, Boult C, Fried LP, Boult L, Wu AW. Clinical practice guidelines and quality of care for older patients with multiple comorbid diseases: implications for pay for performance. JAMA. 2005;294(6):716-24.

18. Polypharmacy in chronic diseases: Reduction of Inappropriate Medication and Adverse drug events in elderly populations by electronic Decision Support (PRIMA-eDS). http://www.prima-eds.eu/. Accessed 20 Aug 2017.

19. Higgins JPT, Green S. Cochrane handbook for systematic reviews of interventions. Version 5.1.0 [updated March 2011]. In.: The Cochrane Collaboration; 2011 Available from: http://handbook-5-1.cochrane.org/. Accessed 20 Aug 2017.

20. Liberati A, Altman DG, Tetzlaff J, Mulrow C, Gotzsche PC, loannidis JP, Clarke M, Devereaux PJ, Kleijnen J, Moher D. The PRISMA statement for reporting systematic reviews and meta-analyses of studies that evaluate healthcare interventions: explanation and elaboration. BMJ. 2009;339:b2700.

21. Martinez YM, Renom-Guiteras A, Reeves D, Ediriweera de Silva RE, Esmail A Kunnamo I, Rieckert A, Sommerauer C, Sönnichsen A. A set of systematic reviews to help reduce inappropriate prescribing to older people: study protocol. BMC Geriatr. 2017;17(1)

22. Shea BJ, Grimshaw JM, Wells GA, Boers M, Andersson N, Hamel C, Porter AC, Tugwell P, Moher D, Bouter LM. Development of AMSTAR: a measurement tool to assess the methodological quality of systematic reviews. BMC Med Res Methodol. 2007;7:10.

23. Shea BJ, Hamel C, Wells GA, Bouter LM, Kristjansson E, Grimshaw J, Henry DA, Boers M. AMSTAR is a reliable and valid measurement tool to assess the methodological quality of systematic reviews. J Clin Epidemiol. 2009;62(10):1013-20.

24. Critical Appraisal Skills Programme: 12 questions to help you make sense of cohort study. In. Oxford; 2013. http://docs.wixstatic.com/ugd/dded87_ 5ad0ece77a3f4fc9bcd3665a7d1fa91f.pdf. Accessed 20 Aug 2017.

25. Critical Appraisal Skills Programme: 11 questions to help you make sense of case control study. In. Oxford; 2013. http://docs.wixstatic.com/ugd/dded87_ afbfc99848f64537a53826e1f5b30b5c.pdf. Accessed 20 Aug 2017.

26. Guyatt GH, Oxman AD, Kunz R, Vist GE, Falck-Ytter Y, Schunemann HJ, Group GW. What is "quality of evidence" and why is it important to clinicians? BMJ. 2008:336(7651):995-8.

27. Guyatt $G H$, Oxman AD, Kunz R, Falck-Ytter $Y$, Vist GE, Liberati $A$ Schunemann HJ, Group GW. Going from evidence to recommendations. BMJ. 2008:336(7652):1049-51.

28. Guyatt GH, Oxman AD, Vist GE, Kunz R, Falck-Ytter Y, Alonso-Coello P, Schunemann HJ. GRADE: an emerging consensus on rating quality of evidence and strength of recommendations. BMJ. 2008;336(7650):924-6.

29. Banerii MA, Purkayastha D, Francis BH. Safety and tolerability of vildagliptin vs. thiazolidinedione as add-on to metformin in type 2 diabetic patients with and without mild renal impairment: a retrospective analysis of the GALIANT study. Diabetes Res Clin Pract. 2010;90(2):182-90.

30. Barzilai N, Guo H, Mahoney EM, Caporossi S, Golm GT, Langdon RB, Williams-Herman D, Kaufman KD, Amatruda JM, Goldstein BJ, et al. Efficacy and tolerability of sitagliptin monotherapy in elderly patients with type 2 diabetes: a randomized, double-blind, placebo-controlled trial. Curr Med Res Opin. 2011;27(5):1049-58.

31. Barnett $A H$, Huisman $H$, Jones $R$, von Eynatten $M$, Patel $S$, Woerle $H J$. Linagliptin for patients aged 70 years or older with type 2 diabetes inadequately controlled with common antidiabetes treatments: a randomised, double-blind, placebo-controlled trial. Lancet. 2013;382(9902): 1413-23.

32. Chien MN, Lee CC, Chen WC, Liu SC, Leung $\mathrm{CH}$, Wang $\mathrm{CH}$. Effect of sitagliptin as add-on therapy in elderly type 2 diabetes patients with inadequate glycemic control in Taiwan. Int J Gerontol. 2011;5(2):103-6.

33. Chang YC, Chuang LM, Lin JW, Chen ST, Lai MS, Chang CH. Cardiovascular risks associated with second-line oral antidiabetic agents added to metformin in patients with type 2 diabetes: a nationwide cohort study. Diabet Med. 2015;32(11):1460-9. 
34. Chen DY, Wang SH, Mao CT, Tsai ML, Lin YS, Su FC, Chou CC, Wen MS, Wang CC, Hsieh IC, et al. Sitagliptin after ischemic stroke in type 2 diabetic patients: a Nationwide cohort study. Medicine. 2015;94(28): e1128.

35. Ou SM, Shih CJ, Chao PW, Chu H, Kuo SC, Lee YJ, Wang SJ, Yang CY, Lin CC, Chen TJ, et al. Effects on clinical outcomes of adding Dipeptidyl peptidase-4 inhibitors versus Sulfonylureas to Metformin therapy in patients with type 2 diabetes mellitus. Ann Intern Med. 2015;163(9):663-72.

36. Shih CJ, Wu YL, Chao PW, Kuo SC, Yang CY, Li SY, Ou SM, Chen YT. Association between use of oral anti-diabetic drugs and the risk of sepsis: a nested case-control study. Sci Rep. 2015;5:15260.

37. Hartley P, Shentu Y, Betz-Schiff P, Golm GT, Sisk CM, Engel SS, Shankar RR. Efficacy and tolerability of Sitagliptin compared with Glimepiride in elderly patients with type 2 diabetes mellitus and inadequate Glycemic control: a randomized, double-blind, non-inferiority trial. Drugs Aging. 2015;32(6):469-76

38. Kadowaki T, Kondo K. Efficacy and safety of teneligliptin added to glimepiride in Japanese patients with type 2 diabetes mellitus: a randomized, double-blind, placebo-controlled study with an open-label, long-term extension. Diabetes Obes Metab. 2014;16(5):418-25.

39. Schernthaner G, Duran-Garcia S, Hanefeld M, Langslet G, Niskanen L, Ostgren CJ, Malvolti E, Hardy E. Efficacy and tolerability of saxagliptin compared with glimepiride in elderly patients with type 2 diabetes: a randomized, controlled study (GENERATION). Diabetes Obes Metab. 2015; 17(7):630-8

40. Schweizer A, Dejager S, Bosi E. Comparison of vildagliptin and metformin monotherapy in elderly patients with type 2 diabetes: a 24-week, doubleblind, randomized trial. Diabetes Obes Metab. 2009;11(8):804-12.

41. Scirica BM, Braunwald E, Raz I, Cavender MA, Morrow DA, Jarolim P, Udell JA, Mosenzon O, Im K, Umez-Eronini AA, et al. Heart failure, saxagliptin, and diabetes mellitus: observations from the SAVOR-TIMI 53 randomized trial. Circulation. 2014;130(18):1579-88.

42. Leiter LA, Teoh H, Braunwald E, Mosenzon O, Cahn A, Kumar KM, Smahelova A, Hirshberg B, Stahre C, Frederich R, et al. Efficacy and safety of saxagliptin in older participants in the SAVOR-TIMI 53 trial. Diabetes Care. 2015;38(6):1145-53.

43. Mosenzon O, Wei C, Davidson J, Scirica BM, Yanuv I, Rozenberg A, Hirshberg B, Cahn A, Stahre C, Strojek K, et al. Incidence of fractures in patients with type 2 diabetes in the SAVOR-TIMI 53 trial. Diabetes Care. 2015;38(11):2142-50.

44. White WB, Cannon CP, Heller SR, Nissen SE, Bergenstal RM, Bakris GL, Perez AT, Fleck PR, Mehta CR, Kupfer S, et al. Alogliptin after acute coronary syndrome in patients with type 2 diabetes. N Engl J Med. 2013;369(14):1327-35.

45. Driessen $\mathrm{JH}$, van Onzenoort HA, Henry RM, Lalmohamed A, van den Bergh JP, Neef C, Leufkens HG, de Vries F. Use of dipeptidyl peptidase-4 inhibitors for type 2 diabetes mellitus and risk of fracture. Bone. 2014;68:124-30.

46. Viljoen A, Meek CL, Gadsby R, Viljoen S, Langerman H, Sinclair AJ. The tolerability and safety of DPP-4 inhibitors for the treatment of older people with type 2 diabetes mellitus: an observational study. Br J Diab Vasc Dis. 2013;13(4):187-91.

47. $\mathrm{Yu} \mathrm{OH}$, Filion $\mathrm{KB}$, Azoulay L, Patenaude $\mathrm{V}$, Majdan A, Suissa S. Incretinbased drugs and the risk of congestive heart failure. Diabetes Care. 2015;38(2):277-84

48. Giorda CB, Picariello R, Tartaglino B, Marafetti L, Di Noi F, Alessiato A, Costa G, Gnavi R. Hospitalisation for heart failure and mortality associated with dipeptidyl peptidase 4 (DPP-4) inhibitor use in an unselected population of subjects with type 2 diabetes: a nested case-control study. BMJ Open. 2015; 5(6):e007959.

49. Penfornis A, Bourdel-Marchasson I, Quere S, Dejager S. Real-life comparison of DPP4-inhibitors with conventional oral antidiabetics as add-on therapy to metformin in elderly patients with type 2 diabetes: the HYPOCRAS study. Diab Metab. 2012;38(6):550-7.

50. Sicras-Mainar A, Navarro-Artieda R. Use of metformin and vildagliptin for treatment of type 2 diabetes in the elderly. Drug Des Dev Ther. 2014;8:811-8.

51. Tziomalos K, Bouziana SD, Spanou M, Kostaki S, Papadopoulou M, Giampatzis V, Dourliou V, Kostourou DT, Savopoulos C, Hatzitolios Al. Prior treatment with dipeptidyl peptidase 4 inhibitors is associated with better functional outcome and lower in-hospital mortality in patients with type 2 diabetes mellitus admitted with acute ischaemic stroke. Diab Vasc Dis Res. 2015;12(6):463-6.
52. Strain WD, Lukashevich V, Kothny W, Hoellinger MJ, Paldanius PM Individualised treatment targets for elderly patients with type 2 diabetes using vildagliptin add-on or lone therapy (INTERVAL): a 24 week, randomised, double-blind, placebo-controlled study. Lancet. 2013;382(9890): 409-16.

53. Schweizer $A$, Dejager $S$. Experience with vildagliptin in patients $>/=75$ years with type 2 diabetes and moderate or severe renal impairment. Diabetes Ther. 2013; 4(2):257-67.

54. Ferrannini E, Fonseca V, Zinman B, Matthews D, Ahren B, Byiers S, Shao Q, Dejager S. Fifty-two-week efficacy and safety of vildagliptin vs. glimepiride in patients with type 2 diabetes mellitus inadequately controlled on metformin monotherapy. Diabetes Obes Metab. 2009;11(2):157-66.

55. Matthews DR, Dejager S, Ahren B, Fonseca V, Ferrannini E, Couturier A, Foley JE, Zinman B. Vildagliptin add-on to metformin produces similar efficacy and reduced hypoglycaemic risk compared with glimepiride, with no weight gain: results from a 2-year study. Diabetes Obes Metab. 2010;12(9):780-9.

56. Fonseca V, Baron M, Shao Q, Dejager S. Sustained efficacy and reduced hypoglycemia during one year of treatment with vildagliptin added to insulin in patients with type 2 diabetes mellitus. Horm Metab Res. 2008;40(6):427-30.

57. Mistry M, Lister N, Andrews C, Geransar P, Sinclair A. A UK retrospective observational survey of vildagliptin as add-on antidiabetes therapy in elderly patients. Br J Diab Vasc Dis. 2011;11(5):239-42.

58. Johansen OE, Neubacher D, von Eynatten M, Patel S, Woerle HJ. Cardiovascular safety with linagliptin in patients with type 2 diabetes mellitus: a pre-specified, prospective, and adjudicated meta-analysis of a phase 3 programme. Cardiovasc Diabetol. 2012;11:3.

59. Rosenstock J, Wilson C, Fleck P. Alogliptin versus glipizide monotherapy in elderly type 2 diabetes mellitus patients with mild hyperglycaemia: a prospective, doubleblind, randomized, 1-year study. Diabetes Obes Metab. 2013;15(10):906-14.

60. Schweizer A, Dejager S, Foley JE, Couturier A, Liqueros-Saylan M, Kothny W. Assessing the cardio-cerebrovascular safety of vildagliptin: meta-analysis of adjudicated events from a large phase III type 2 diabetes population. Diabetes Obes Metab. 2010;12(6):485-94.

61. Weir DL, McAlister FA, Senthilselvan A, Minhas-Sandhu JK, Eurich DT. Sitagliptin use in patients with diabetes and heart failure: a populationbased retrospective cohort study. JACC Heart Fail. 2014;2(6):573-82.

62. Wu S, Hopper I, Skiba M, Krum H. Dipeptidyl peptidase-4 inhibitors and cardiovascular outcomes: meta-analysis of randomized clinical trials with 55,141 participants. Cardiovasc Ther. 2014;32(4):147-58.

63. Egan AG, Blind $E$, Dunder $K$, de Graeff PA, Hummer BT, Bourcier $T$, Rosebraugh C. Pancreatic safety of incretin-based drugs-FDA and EMA assessment. N Engl J Med. 2014;370(9):794-7.

64. Gerstein HC, Miller ME, Genuth S, Ismail-Beigi F, Buse JB, Goff DC Jr, Probstfield JL, Cushman WC, Ginsberg HN, Bigger JT, et al. Long-term effects of intensive glucose lowering on cardiovascular outcomes. N Engl J Med. 2011;364(9):818-28

65. American Geriatrics Society Expert Panel on Care of Older Adults with Diabetes M, Moreno G, Mangione CM, Kimbro L, Vaisberg E. Guidelines abstracted from the American Geriatrics Society guidelines for improving the Care of Older Adults with diabetes mellitus: 2013 update. J Am Geriatr Soc. 2013;61(11):2020-6.

66. International Diabetes Federation: International diabetes federation. Managing older people with type 2 diabetes. Global guideline. In. Brussels, Belgium: International Diabetes Federation; 2013. https://www.idf.org/elibrary/guidelines/78-global-guideline-for-managing-older-people-with-type2-diabetes.html. Accessed 20 Aug 2017.

67. Sinclair AJ, Paolisso G, Castro M, Bourdel-Marchasson I, Gadsby R, Rodriguez Manas L, European Diabetes Working Party for Older P. European diabetes working Party for Older People 2011 clinical guidelines for type 2 diabetes mellitus. Executive summary. Diab Metab. 2011;37(Suppl 3):S27-38.

68. Sinclair A, Morley JE, Rodriguez-Manas L, Paolisso G, Bayer T, Zeyfang A, Bourdel-Marchasson I, Vischer U, Woo J, Chapman I, et al. Diabetes mellitus in older people: position statement on behalf of the International Association of Gerontology and Geriatrics (IAGG), the European diabetes working Party for Older People (EDWPOP), and the international task force of experts in diabetes. J Am Med Dir Assoc. 2012;13(6):497-502.

69. Onder G, Landi F, Fusco D, Corsonello A, Tosato M, Battaglia M, Mastropaolo S, Settanni S, Antocicco M, Lattanzio F. Recommendations to prescribe in complex older adults: results of the CRIteria to assess appropriate medication use among elderly complex patients (CRIME) project. Drugs Aging. 2014;31(1):33-45 
70. Wieczorek A, Rys P, Skrzekowska-Baran I, Malecki M. The role of surrogate endpoints in the evaluation of efficacy and safety of therapeutic interventions in diabetes mellitus. Rev Diab Stud. 2008;5(3):128-35.

71. Holleman F, Uijldert M, Donswijk LF, Gale EA. Productivity of authors in the field of diabetes: bibliographic analysis of trial publications. BMJ. 2015;351:h2638.

72. Food US, Administration D. FDA approves new treatment for diabetes. Maryland: US Food and Drug Administration; 2006

73. Elwyn G, Frosch D, Thomson R, Joseph-Williams N, Lloyd A, Kinnersley P, Cording E, Tomson D, Dodd C, Rollnick S, et al. Shared decision making: a model for clinical practice. J Gen Intern Med. 2012;27(10):1361-7.

74. FDA U.S. Food and Drug Administration. Diabetes Medications Containing Saxagliptin and Alogliptin: Drug Safety Communication - Risk of Heart Failure. http:/wwww.fda.gov/Safety/MedWatch/Safetylnformation/SafetyAlertsforHuman MedicalProducts/ucm494252.htm. Accessed 20 Aug 2017.

\section{Submit your next manuscript to BioMed Central} and we will help you at every step:

- We accept pre-submission inquiries

- Our selector tool helps you to find the most relevant journal

- We provide round the clock customer support

- Convenient online submission

- Thorough peer review

- Inclusion in PubMed and all major indexing services

- Maximum visibility for your research

Submit your manuscript at www.biomedcentral.com/submit 